\title{
Urogenital tuberculosis - epidemiology, pathogenesis and clinical features
}

Asif Muneer ${ }^{1}$, Bruce Macrae ${ }^{2}$, Sriram Krishnamoorthy ${ }^{3}$ and Alimuddin Zumla ${ }^{2,4,5 *}$

Abstract | Tuberculosis (TB) is the most common cause of death from infectious disease worldwide. A substantial proportion of patients presenting with extrapulmonary TB have urogenital TB (UG-TB), which can easily be overlooked owing to non-specific symptoms, chronic and cryptic protean clinical manifestations, and lack of clinician awareness of the possibility of TB. Delay in diagnosis results in disease progression, irreversible tissue and organ damage and chronic renal failure. UG-TB can manifest with acute or chronic inflammation of the urinary or genital tract, abdominal pain, abdominal mass, obstructive uropathy, infertility, menstrual irregularities and abnormal renal function tests. Advanced UG-TB can cause renal scarring, distortion of renal calyces and pelvic, ureteric strictures, stenosis, urinary outflow tract obstruction, hydroureter, hydronephrosis, renal failure and reduced bladder capacity. The specific diagnosis of UG-TB is achieved by culturing Mycobacterium tuberculosis from an appropriate clinical sample or by DNA identification. Imaging can aid in localizing site, extent and effect of the disease, obtaining tissue samples for diagnosis, planning medical or surgical management, and monitoring response to treatment. Drug-sensitive TB requires $6-9$ months of WHO-recommended standard treatment regimens. Drug-resistant TB requires $12-24$ months of therapy with toxic drugs with close monitoring. Surgical intervention as an adjunct to medical drug treatment is required in certain circumstances. Current challenges in UG-TB management include making an early diagnosis, raising clinical awareness, developing rapid and sensitive TB diagnostics tests, and improving treatment outcomes.

Tuberculosis (TB) is among the most common causes of death from infectious disease worldwide ${ }^{1}$. The WHO Global Tuberculosis Report 2018 (REF. ${ }^{2}$ ) states that in 2017 an estimated 10 million people (5.8 million men, 3.2 million women and 1 million children) developed TB and 4 million people with TB remained undiagnosed and untreated. Of the 10 million people with TB, two-thirds were in eight countries: India (27\%), China (9\%), Indonesia (8\%), the Philippines (6\%), Pakistan (5\%), Nigeria (4\%), Bangladesh (4\%) and South Africa (3\%). Only $6 \%$ of global incidences were in the WHO European Region (3\%) and the WHO Region of the Americas (3\%). Overall, 464,633 people with HIV also had TB, of whom $72 \%$ were from Africa. TB occurs in all countries and age groups, with $90 \%$ of infections being reported in adults (aged $\geq 15$ years). About 558,000 people developed rifampicin-resistant $\mathrm{TB}$, of whom an estimated 458,000 had multidrug-resistant TB (MDR-TB) (defined as resistance to two first-line drugs, rifampicin and isoniazid) ${ }^{2}$. Only $50 \%$ of patients with MDR-TB are cured after treatment with WHO-approved regimens $s^{3,4}$. $\mathrm{TB}$ can affect any part of the body ${ }^{5,6}$. Of the 10 million annual incidences of TB, between $5 \%$ and $45 \%$ have features of extrapulmonary TB (EPTB) ${ }^{5-7}$ affecting all organs of the body. Common sites of EPTB are lymph nodes, pleura, bones, meninges and the urogenital tract. TB affecting the kidneys, ureters, bladder, prostate, urethra, penis, scrotum, testicles, epididymis, vas deferens, ovaries, fallopian tubes, uterus, cervix and vulva were initially grouped together as genitourinary $\mathrm{TB}^{8-11}$. Currently, the term urogenital TB (UG-TB) is thought to be more appropriate as urinary tract $\mathrm{TB}$ occurs more often than genital $\mathrm{TB}^{10}$.

UG-TB is a neglected clinical problem and can easily be overlooked owing to non-specific symptoms, chronic and cryptic protean clinical manifestations, and a lack of clinician awareness of the possibility of $\mathrm{TB}^{11-26}$. A delay in making a diagnosis results in disease progression, ureteral strictures, contracted bladder, obstructive nephropathy, renal parenchymal destruction, irreversible organ damage and end-stage renal failure ${ }^{10}$. In this Review, we describe the epidemiology, pathogenesis, clinical features, diagnosis and management of UG-TB. 


\section{Key points}

- Between $15 \%$ and $40 \%$ of the 10 million new patients diagnosed with tuberculosis (TB) annually present with extrapulmonary TB (EPTB), of which a considerable proportion have urogenital TB (UG-TB). Patients who have had a renal transplant, have HIV infection, receive immunosuppressive therapies, have diabetes, have COPD and those undergoing dialysis often experience reactivation of latent TB infection.

- UG-TB is often missed clinically or is diagnosed late, owing to the lack of awareness among clinicians, its insidious onset, chronic non-specific symptoms, and cryptic and protean clinical manifestations, resulting in disease progression.

- Specific diagnosis of TB is made by identification of Mycobacterium tuberculosis (Mtb) in clinical samples, by microscopy and culture, or by identification of Mtb DNA. Imaging can aid in identifying disease sites and obstructive lesions, guiding biopsies and surgical interventions

- Treatment of drug-sensitive TB requires 6-9 months of the WHO-recommended standard treatment regimen, but longer therapy is needed for severe disease or in patients in whom immunosuppression is an underlying risk factor. Multidrug-resistant TB requires between 12 and 24 months of therapy with toxic drugs and careful monitoring.

- Surgery is indicated for complications of UG-TB. Nephrectomy is required for severely damaged kidneys, and reconstruction procedures include pyeloureteral anastomosis, ureterocalyceal anastomosis, caliceal reconstruction, uretero-ureteral anastomosis and ureter substitution by ileum.

\section{Epidemiology}

TB is caused by bacilli of the Mycobacterium tuberculosis complex (MTBC) $)^{5,627-29}$. These bacilli include Mycobacterium tuberculosis (Mtb), Mycobacterium bovis, Mycobacterium africanum (which causes human TB in West and East Africa), Mycobacterium caprae, Mycobacterium pinnipedii, Mycobacterium microti and bacillus Calmette-Guérin (BCG), the derivative of $M$. bovis used in vaccines. $M t b$ and $M$. africanum are the most frequent causes of human TB causing an estimated $98 \%$ of infections. M. bovis is the next most common cause of $\mathrm{TB}$ and is responsible for an estimated $1.8 \%$ of cases $^{30}$.

Accurate epidemiological and clinical data on UG-TB are difficult to obtain owing to challenges in making an accurate diagnosis, protean and non-specific clinical manifestations, a lack of clinical awareness of the possibility of TB, presence of other comorbidities such as HIV, diabetes and bacterial urinary tract infection (UTI $)^{5,6}$. Thus, the exact prevalence of UG-TB in various geographical locations and specific patient groups is difficult to estimate because a considerable number of patients remain asymptomatic and undiagnosed. The proportion of UG-TB among all forms of EPTB reported in the literature varies according to geographical region, from $15-20 \%$ in Africa, Asia, eastern Europe and the Russian Federation to $2-10 \%$ in western Europe and the USA. Variation between geographical areas might reflect local endemic TB prevalence rates or study bias.

\footnotetext{
Author addresses

${ }^{1}$ Department of Urology, and NIHR Biomedical Research Centre, University College

London Hospitals NHS Foundation Trust, London, UK.

2Department of Clinical Microbiology, University College London Hospitals NHS

Foundation Trust, London, UK.

${ }^{3}$ Department of Urology \& Renal transplantation, Sri Ramachandra Medical College $\mathcal{E}$

Research Institute, Chennai, Tamil Nadu, India.

${ }^{4}$ Division of Infection and Immunity, Centre for Clinical Microbiology, University College London, London, UK.

${ }^{5}$ NIHR Biomedical Research Centre, UCL Hospitals NHS Foundation Trust, London, UK.
}

UG-TB can remain subclinical ${ }^{31}$ and, therefore, current data are only estimates ${ }^{10,13,23,32}$. UG-TB can occur concurrently in up to $20 \%$ of individuals with pulmonary TB (PTB) $)^{10,13-25,32,33}$.

Autopsy studies ${ }^{34-37}$ provide insights into the natural history and pathogenesis of UG-TB. UG-TB was found in $3.1 \%$ of 5,424 autopsies studied ${ }^{34}$. Of these people, urogenital involvement was bilateral in $98 \%$ and $85 \%$ had concomitant pulmonary lesions. Autopsy records of 200 children and 92 young adults $<25$ years of age who died of TB showed that $65 \%$ of renal TB lesions were miliary and $23 \%$ were caseous ${ }^{36}$. An autopsy study from India of 35 patients with AIDS identified 17 cases of renal $\mathrm{TB}^{37}$. Another autopsy study of 87 people in Mexico City showed that of 36 people with any type of kidney infection, 19 had $M t b^{38}$, indicating that the diagnosis of urological TB is often missed antemortem and is only detected at autopsy.

\section{Risk factors}

Risk factors for developing TB include malnutrition, HIV infection, diabetes, chronic renal and liver disease, alcohol and substance abuse, smoking, homelessness, poor housing, pneumoconiosis, genetics, vitamin deficiency, immunosuppressive drugs, renal transplantation, chronic renal disease, dialysis and end-stage renal failure $^{5,6}$. The frequency of UG-TB seen in clinical practice varies according to age, gender, geographical region, HIV prevalence in the community, immunosuppressive therapy and comorbidities ${ }^{10,13,39-45}$. UG-TB is reported to affect twice as many women as men ${ }^{13,32,40}$, but this estimate is controversial owing to the lack of controlled epidemiological and clinical studies. Increased rates of TB are seen in patients who have had a kidney transplant, patients with end-stage renal disease and those undergoing peritoneal dialysis ${ }^{46-52}$.

\section{Mode of transmission}

Mycobacteria of the MTBC can be transmitted to humans in several ways (TABLE 1). The most common routes involve person-to-person transmission through inhalation of $M t b$-infected droplet aerosols from the coughs or sneezes of people with active pulmonary TB and ingestion of M. bovis-infected raw unpasteurized dairy products $27,30,53$.

Other rare modes of $M t b$ infection that have been reported include congenital transmission, sexual transmission, accidental inoculation, vaccination and therapeutic instillation ${ }^{54-72}$. Postulated modes of congenital and neonatal transmission include transplacental transmission via blood or lymphatics from a mother with active TB or aspiration or ingestion of $M t b$-infected amniotic fluid during birth ${ }^{54-60}$.

Sexual transmission of $M t b$ has been reported in a study that found the $M t b$ molecular subtype isolated from skin ulcers on the penis of a husband was identical to the subtype isolated from his wife who had endometrial $\mathrm{TB}^{61} . M t b$ has also been isolated from the ejaculate of men with TB of the prostate ${ }^{62-64}$.

Vaccination with live BCG vaccine of HIV-infected and immunosuppressed individuals has resulted in local and disseminated BCG M. bovis strain $\mathrm{TB}^{65,66}$. 
Table 1 | Routes, sources and modes of Mycobacterium tuberculosis complex transmission

\begin{tabular}{|c|c|c|}
\hline Route of transmission & Mode of transmission and source of infection & Refs \\
\hline \multicolumn{3}{|l|}{ Common routes } \\
\hline $\begin{array}{l}\text { Inhalation (airborne) } \\
\text { ( }>95 \% \text { cases) }\end{array}$ & Inhalation of Mtb-infected droplets from cough of patients with active pulmonary TB & $5,6,53$ \\
\hline Oral (ingestion) & $\begin{array}{l}\text { Consumption of dairy products infected with Mycobacterium bovis from cattle with } \\
\text { active bovine TB }\end{array}$ & $27,28,30$ \\
\hline \multicolumn{3}{|l|}{ Uncommon routes } \\
\hline Congenital or neonatal & $\begin{array}{l}\text { Possible mechanisms: transplacental transmission; via bloodstream or lymphatics } \\
\text { from mother with active TB disease; directly from placenta with miliary TB; or } \\
\text { aspiration or ingestion of Mtb-infected amniotic fluid during birth }\end{array}$ & $54-59$ \\
\hline Parenteral (injection) & $\begin{array}{l}\text { Intravesical instillation of live BCG M. bovis vaccine strain as adjuvant treatment } \\
\text { of carcinoma in situ of the bladder leads to local or invasive disease (bladder, } \\
\text { epididymis, penis, prostate and renal TB have been described); BCG vaccination in } \\
\text { HIV-infected (immunosuppressed) individuals causes disseminated BCG disease }\end{array}$ & $65,67-72$ \\
\hline Sexual & $\begin{array}{l}\text { Direct contact with active genital TB lesions or exudates containing } M t b \text {; sexual } \\
\text { transmission of } M t b \text { (Mtb has been isolated from semen of men with TB of the prostate) }\end{array}$ & $61,62,81,305$ \\
\hline
\end{tabular}

Intravesical instillation of BCG as adjuvant treatment for transitional cell carcinoma in situ of the bladder is widely practised. TB of the bladder, epididymis, prostate and kidney have been reported following intravesical instillation with BCG for bladder cancer ${ }^{67-72}$. Patients receiving this treatment should, therefore, be counselled beforehand regarding this risk.

\section{Pathogenesis of UG-TB}

The pathogenesis of $M t b$ in humans has been extrapolated from animal models, as tissue from people with untreated TB is lacking and limits our understanding of UG-TB ${ }^{73,74}$. The exact sequence of events following primary infection (the first infection in a previously unexposed individual) in humans with any mycobacterial species of the MTBC remains unknown ${ }^{75-78}$. Some people are naturally resistant to $M t b$ infection and are able to eradicate the mycobacteria owing to innate immunity $^{79}$. Following primary infection through inhalation or ingestion, the $M t b$ bacilli (FIG. 1) multiply locally in tissues and evoke a complex array of immune responses, which result in either elimination (eradication) or containment via primary granuloma formation (the primary Ghon focus). Primary TB lesions are found in the lungs, tonsils or gut, but any organ can harbour the primary focus. Rare cases of primary genital TB of the cervix, vagina or vulva have been reported in women ${ }^{80}$ whose male partners had active UG-TB or pulmonary TB, transmission being assumed through infected semen ${ }^{81,82}$ or sputum used as a sexual lubricant ${ }^{83}$. TB of the penis in infants has been reported after ritual circumcision ${ }^{61,84}$.

Histologically, primary granulomas consist of a compact focal collection of inflammatory and immune cells such as neutrophils, T lymphocytes and B lymphocytes, epithelioid cells, macrophages, Langhans giant cells, and fibroblasts, with central caseous necrosis ${ }^{75,85}$. If not contained at this stage, the mycobacteria spread via the lymphatics (causing lymphangitis) and to regional lymph nodes (causing lymphadenitis). Lymph nodes can undergo caseous necrosis and some nodes might coalesce together with time to form a whole mass ${ }^{75}$. In the lungs or in the gut, the triad of the primary Ghon focus, lymphangitis and lymphadenitis are together known as the primary Ghon complex (FIG. 2).

Epidemiological and longitudinal cohort studies have indicated that in the majority of people (95\%) with primary $M t b$ infection, the mycobacteria are eventually either eliminated or contained as latent TB infection (LTBI) $)^{5,6,79}$. Progression of the primary disease occurs in $5-10 \%$ of people, leading to local spread to adjacent tissues or widespread systemic dissemination of mycobacteria via the bloodstream and lymphatics (FIG. 2). Seeding of tissues of all organs of the body outside the lung can occur, known as EPTB. EPTB includes infection of all tissues of the urological and reproductive tracts. Mycobacteria in these organs can progress to cause disease over time (known as progressive primary TB) or can be contained as LTBI. A proportion of people with EPTB do not show signs of disease and continue to have subclinical $\mathrm{TB}^{6,31}$.

The slow replication rate of $M t b$, its intracellular location in macrophages and acquired immune responses ${ }^{79}$ means it takes between 12 months and 2 years following primary infection for symptoms and signs of the disease to manifest ${ }^{27,29}$. The constant chronic interplay between $M t b$ and the host immune response can lead to eradication of $M t b$, or progression of disease manifesting with caseous necrosis, miliary disease, formation of abscesses, cysts, ulcers, fistulae, fibrosis or calcification. Immunosuppression reduces cell-mediated immune responses and allows proliferation of $M t b$ bacilli, causing severe disease that progresses rapidly ${ }^{4}$.

An estimated 1.7 billion people (one-quarter of the world's population) have LTBI and have no symptoms or signs of disease ${ }^{5,6,86}$. LTBI is defined by WHO as a state of persistent immune response to stimulation by $M t b$ antigens with no evidence of clinically manifest active $\mathrm{TB}^{2}$. As people with LTBI have viable bacilli within their tissues, they are at risk of reactivation into active TB disease. LTBI will reactivate in 5-15\% of people and progress to active $\mathrm{TB}^{5,6,76-78,87}$, adding to the pool of infectious people worldwide. In these individuals, the period between 
latency following primary infection and reactivation at a later stage in life ranges from 1 year to 50 years ${ }^{86,87}$. UG-TB can present in any age group, but, owing to the long latency period, it commonly affects those aged $30-50$ years and is infrequently reported in children ${ }^{45,88}$.

\section{Urogenital spread}

After primary $M t b$ infection at any organ site, direct haematogenous or lymphatic spread from primary $M t b$ lesions leads to $M t b$ seeding into various parts of the urogenital tract (FIG. 2). Mtb from the kidney can enter

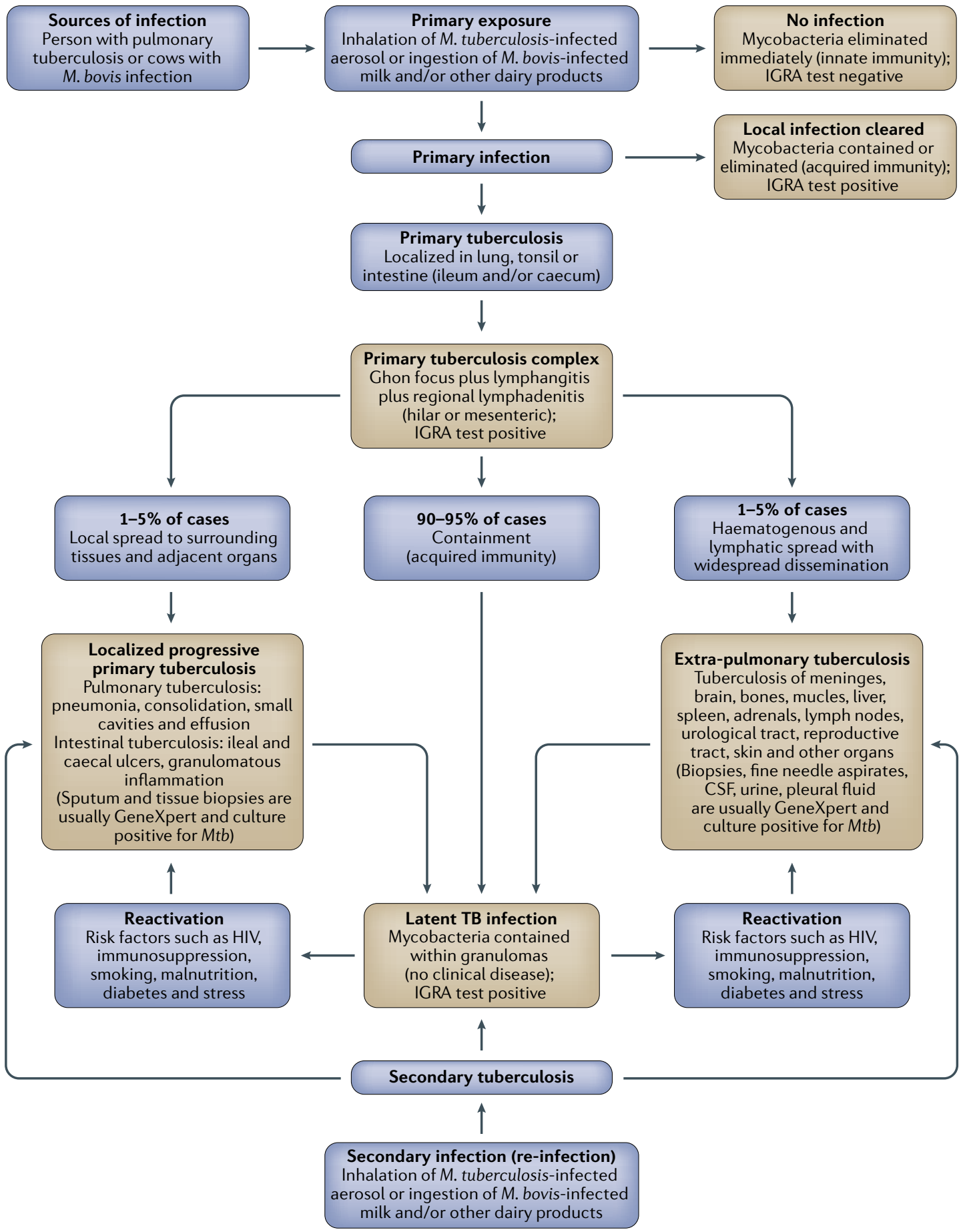

Fig. 1 | Pathogenesis and natural history of Mycobacterium tuberculosis infection. The pathogenesis of Mycobacterium tuberculosis (Mtb) in humans has been extrapolated from animal models. The exact sequence of events following primary infection in humans with any mycobacterial species of the Mycobacterium tuberculosis complex (MTBC) remains unknown. Following primary infection through inhalation or ingestion, the Mtb bacilli evoke a complex array of immune responses, which result in either elimination (eradication) or containment via primary granuloma formation (the primary Ghon focus). Some people are naturally resistant to $M t b$ infection and are able to eradicate the mycobacteria owing to innate immunity. CSF, cerebrospinal fluid; IGRA, interferon- $\gamma$ release assay; TB, tuberculosis. 
and lodge in the urothelium, ureter, bladder, urethra, seminal vesicles and testes ${ }^{13,34,35}$. A study of 5,424 autopsies performed on men (>16 years) published in 1949 found that 153 men had renal TB, of whom 50 had genital involvement, suggesting spread of $M t b$ in urine from the kidney to the collecting system and into the renal pelvicalyceal system and flowing downstream ${ }^{35}$. In this autopsy study, no TB lesions were observed in the kidneys of $11 \%$ of men with genital TB, indicating that direct local extension from adjacent foci into the bloodstream and lymphatics leads to haematogenous or lymphatic spread and that all mechanisms of spread might work in tandem. As the disease progresses, concurrent, sequential or progressive disease in various urogenital sites occurs long before TB is diagnosed clinically $^{13-19}$. Clinical and autopsy studies show that unilateral involvement is common, but bilateral disease and combined involvement of the kidney, prostate, seminal vesicles and epididymis can also occur ${ }^{32,35,36}$. A large percentage of UG-TB can remain subclinical ${ }^{31}$.

\section{Renal TB}

Renal TB (FIG. 3) is the most frequently diagnosed clinical presentation of UG-TB ${ }^{13,19,20,32,46,89-93}$. Up to $10 \%$ of patients with renal TB have active pulmonary TB and the chest X-ray is abnormal in $50 \%$ of patients with evidence of previous $\mathrm{TB}^{13,34}$. The kidneys are highly vascularized organs and both kidneys are usually seeded with mycobacteria through haematogenous or lymphatic $\operatorname{spread}^{34,36,94,95}$. Thus, $M t b$-induced granulomas and granulation of tissue with caseous necrosis can occur throughout the renal tissue (FIG. 3a,b). These features are seen particularly in the cortex, adjacent to glomeruli (FIC. 3c) or the peritubular capillary bed (FIG. 3d). Miliary microscopic tubercles enlarge and coalesce, becoming macroscopically visible in the upper and lower poles of the renal cortex as pale yellow or white lesions up to $3 \mathrm{~mm}$ in diameter (FIG. 3a,b). In HIV-infected or other immunosuppressed patients, the granulomas are less-well formed, kidney disease is diffuse and lymph node involvement with numerous $M t b$ bacilli is present ${ }^{21,37,96}$.

Granulomatous inflammation and disease progression leads to chronic tubulointerstitial nephritis, papillary necrosis, ulcers, fibrosis with extensive caseous destruction of the renal parenchyma and formation of lobules (FIG. 3e), dilated calyces (FIG. 3f,g) and cavities ${ }^{19,75,88,97}$ (FIG. 3h). Extensive areas of papillary necrosis form cavities and cause vascular insufficiency in renal papillae, leading to papillary necrosis. The dissemination of infection to the renal pelvis can cause tuberculous pyelonephritis, which can evolve into pyonephrosis with progressive fibrosis and scarring of the renal pelvis and ureteropelvic junction, leading to urinary flow obstruction and dilated calyces. These processes evolve over several years. Extensive necrosis replaces the kidney parenchyma with caseous material (called putty kidney) (FIG. 3i). In 20-40\% of instances of renal TB, varying degrees of ill-defined, irregular renal parenchymal calcification occur ${ }^{98,99}$, which are seen on imaging (FIG. $3 j-1$ ) and at surgery (FIG. 3m,n). According to the extent of tissue destruction, kidney TB (KTB) pathology can be classified into four stages: stage 1 (KTB-1; non-destructive form) refers to TB of the kidney parenchyma; stage 2 (KTB-2; small destructive form) refers to TB papillitis; stage 3 (KTB-3; destructive form), refers to cavernous kidney TB; and stage 4 (KTB-4; widespread destructive form) is polycavernous kidney $\mathrm{TB}^{100}$. With bilateral disease, glomerular filtration rate (GFR) and renal function decline progressively, causing end-stage renal failure ${ }^{101-104}$. Non-functioning kidneys might eventually require nephrectomy ${ }^{13,14,19,105,106}$. Renal disease can also extend into the psoas sheath and the perirenal and pararenal spaces to form cold abscesses, sinus tracts and fistulae ${ }^{107-109}$.

Renal TB is an underdiagnosed and important clinical problem. The kidney is usually infected with $M t b$ by haematogenous spread from the lung or gut. The symptoms, signs and imaging features are non-specific. Untreated, disease progression leads to destruction of the renal parenchyma and obstructive nephropathy with end-stage renal failure.

\section{TB of the ureters}

$\mathrm{TB}$ of the ureters can involve any part of the ureter, although the lower third is the most frequently affected site followed by the ureteropelvic junction ${ }^{110}$. Ureteric involvement per se without renal TB has not been described. Up to $50 \%$ of patients with renal TB have ureteric involvement $^{13,14,32,34,91}$. Mtb bacilli from renal medullary lesions spread downstream with the urine to the ureters, the ureterovesical junction and into the bladder ${ }^{10-12,91}$. Ureteric involvement leads to inflammation, oedema, granulomatous ulceration and fibrosis resulting in irregular ureteric strictures, segmental dilation, with ureteric obstruction and reflux ${ }^{100,107,108}$. Ureteritis follows mucosal involvement with the formation of granulomas in the ureteric wall. Intravenous urography (IVU) or retrograde pyelography might show mucosal granulomas as intraluminal filling defects with mucosal ulceration showing up as irregularities in the ureter ${ }^{107,108,110,111}$. Chronic inflammation and ureteric strictures lead to progressive hydroureteronephrosis (FIGS 4,5). Alternating areas of non-confluent dilatations and strictures can appear as a corkscrew or beaded configuration; furthermore, irregularity of the mucosa, dilation and stricture formation (called sawtooth ureter), ureteral shortening and rigid fibrotic ureter lacking peristaltic movement (pipe-stem ureter) ${ }^{112}$ can occur. Progression of disease can eventually lead to the ureter becoming a shortened and rigid tube $\mathrm{e}^{100-112}$. Ureteric calcification can occur and differentiation from ureteric schistosomiases is required ${ }^{113}$.

Involvement of the ureters is frequent in people with renal TB and occurs downstream of $M t b$ infection of the kidney. Ureteric stricture with obstructive uropathy is an important complication that needs to be differentiated from other causes of stricture.

\section{Tuberculosis of the bladder}

Bladder TB (FIG. 5) usually occurs secondary to renal TB with $M t b$ entering the urine and the bladder and occurs in up to $21 \%$ of patients ${ }^{13}$. Spread through lymphatics and blood vessels from primary or secondary TB lesions elsewhere and retrograde spread of $M t b$ from testicular or prostatic TB can also occur ${ }^{10,13}$. Primary bladder TB has also been described in patients with 
Routes of infection:

- Inhalation of aerosols containing M. tuberculosis ( from a pa pulmonary TB - -

- Ingestion of M. bovis
in dairy products $-1$

4 Fig. 2 | Mycobacterium tuberculosis infection. Seeding of tissues of all organs of the body can occur, which is known as extrapulmonary tuberculosis (EPTB). EPTB includes infection of all tissues of the urological and reproductive tracts. Routes of infection include inhalation of aerosols containing Mycobacterium tuberculosis (Mtb) or ingestion of Mycobacterium bovis in dairy products. Kidneys can be seeded by haematogenous spread, lymphatic spread or spread after primary infection from the lungs or intestines. $M t b$-induced granulomas and granulation tissue with caseous necrosis are particularly seen in renal tuberculosis (TB), adjacent to glomeruli and in the peritubular capillary bed. Bladder infection can occur secondary to kidney infection, in which Mtb descends via the urine, or through haematogenous or lymphatic spread. TB of the bladder has also been shown to occur via retrograde spread of TB from the prostate or testes. Prostatic TB can occur via haematogenous or lymphatic spread from pulmonary TB or local spread from epididymal TB. TB of the testes, epididymis, vas deferens and seminal vesicles occurs via haematogenous spread or retrograde spread from the prostate via the vas, peri-vas lymphatics or capillaries. Infection of the female genital tract occurs via haematogenous or lymphatic spread from primary or secondary pulmonary TB.

bladder carcinoma in situ treated with intravesical BCG vaccine instillations $s^{67,69,70,72}$.

Bladder TB can present as cystitis ${ }^{114}$ that consists of a superficial granulomatous inflammation and oedematous swelling of the mucosal surface, which can be focal or generalized across the entire bladder. Tuberculomas of the bladder wall can form, seen as filling defects on imaging ${ }^{107,108}$. Chronic inflammation at the ureterovesical junction can lead to progressive fibrosis, narrowing, stenosis and stricture formation (FIG. 5a), scarification (often described as a golf-hole appearance) of the ureteric orifice $^{10,107,108,110,114}$, and consequential ureterovesical reflux (FIG. 5b,c) and hydroureteronephrosis. Chronic inflammation of the bladder wall and detrusor muscle can lead to reduction in the bladder capacity (thimble bladder) (FIG. 5 d) owing to progressive thickening of the bladder wall with trabeculation and calcification ${ }^{19,107,108}$. Fibrosis in the region of the trigone produces gaping of the ureterovesical junction, resulting in ureterovesical $\operatorname{reflux}^{10,107,108}$. Rare complications of bladder TB include vesicovaginal, vesicocolic and enterovesical fistulae and bladder perforation ${ }^{100,107,108,115}$. TB of the bladder can be classified into four stages ${ }^{100}$ : stage 1 (tubercle-infiltrative bladder TB); stage 2 (erosive-ulcerous bladder TB); stage 3 (interstitial cystitis/painful bladder syndrome); and stage 4 (contracted bladder up to full obliteration). $\mathrm{TB}$ cystitis is indistinguishable from other infectious aetiologies ${ }^{100}$. TB should always be a differential diagnosis when patients with recurrent UTI fail to respond to antibiotic therapy ${ }^{115}$ or are being investigated for cancer or other granulomatous disorders of the bladder, such as helminth infections, and vice versa ${ }^{13,85,116,117}$.

Bladder TB can arise from urinary spread of $M t b$ from the upper urinary tract or from retrograde spread from the prostate or testicles. TB cystitis should be considered in the differential diagnosis when patients are investigated for bladder cancer or granulomatous diseases in the bladder. Long-term bladder TB can result in the development of strictures at the ureteric orifice ${ }^{110}$ or a contracted bladder ${ }^{13,107,108,113}$.

\section{Prostatic TB}

$M t b$ infection of the prostate can occur through haematogenous or lymphatic spread from pulmonary and renal TB or local spread from epidydimal $\mathrm{TB}^{118-123}$. Thus, prostatic TB can commonly coexist with kidney TB and
TB epididymo-orchitis ${ }^{118}$. Prostatic TB has been known to develop after intravesical BCG therapy for bladder cancer $^{71}$. The presence of $M t b$ in semen in some men has raised the possibility of sexual transmission ${ }^{81,82}$.

$M t b$ infection of the prostate leads to chronic inflammation and caseous necrosis with formation of cavities and abscesses, which can drain into surrounding tissues with fistulae formation in the perineum (FIG. 6), urethra or scrotum ${ }^{118,122-129}$. Urine flow through multiple urethral, perineal or rectal fistulae is referred to as the watering-can effect ${ }^{123}$.

Prostatic TB can coexist with renal TB and TB epididymo-orchitis. Chronic TB of the prostate can result in the development of fistulae in the urethra, perineum and scrotum owing to chronic inflammation and caseous necrosis.

\section{Scrotal and seminal vesicle tuberculosis}

Scrotal TB refers to TB of the testis, epididymis (FIG. 7) and vas deferens ${ }^{125-138}$. A study of 29 patients with scrotal TB showed that $25 \%$ had evidence of active pulmonary $\mathrm{TB}^{129}$. In these patients, the mean interval from emergence of symptoms to making a clinical diagnosis was 142 days, reflecting the long delay in making a diagnosis of TB.

$\mathrm{TB}$ of the seminal vesicles and vas deferens occurs secondary to prostate TB and can cause infertility ${ }^{129-131,138}$. $\mathrm{TB}$ of the testis occurs secondary to $\mathrm{TB}$ of the epididymis, which has an extensive blood supply and acquires $M t b$ infection secondary to haematogenous spread of $M t b$ bacilli. $M t b$ bacilli transit along the vas or through the perivasal lymphatics to the epididymis ${ }^{129-134}$. In adults, TB epididymo-orchitis is caused by direct spread from the lower urinary tract or retrograde spread of $M t b$ bacilli via the prostate and into the seminal vesicles, vas and epididymis ${ }^{131-133}$. Observations of isolated epididymal TB lesions in children suggest the possibility of haematogenous spread ${ }^{132,133}$. TB-induced orchitis following intravesical BCG therapy can occur ${ }^{69,70,72}$.

The epididymis is a common site of involvement and can be the first or the only presenting feature of UG-TB in men ${ }^{130,135-139}$. Up to $50 \%$ of men with TB epididymo-orchitis initially have involvement of the epididymis alone, with testicular involvement developing later as the disease progresses ${ }^{32,135}$. Epididymal TB presents with co-involvement of the prostate in up to $39 \%$ of patients ${ }^{13,130,132,138}$. The cauda of the epididymis is affected more than the caput, possibly owing to the increased vascularity of the cauda ${ }^{136}$. Spermatic cord tuberculoma has been described and mimics a testicular tumour ${ }^{140}$. Nodular beading of the vas deferens or a dilated epididymis are characteristic physical findings and cause obstructive azoospermia ${ }^{139}$. Direct involvement of the epididymis resulting in occlusion of the tubules and ejaculatory ducts caused by scarring and distortion of the normal anatomy can cause infertility ${ }^{140}$. TB of the seminal vesicles can result in calculi or abscess formation ${ }^{141}$.

The vascularity of the epididymis allows haematogenous spread of $M t b$ to the scrotal contents; retrograde transmission to the prostate and seminal vesicle results in coexisting disease in the prostate. The development of strictures in the vas deferens and ejaculatory ducts results in men developing obstructive azoospermia. 

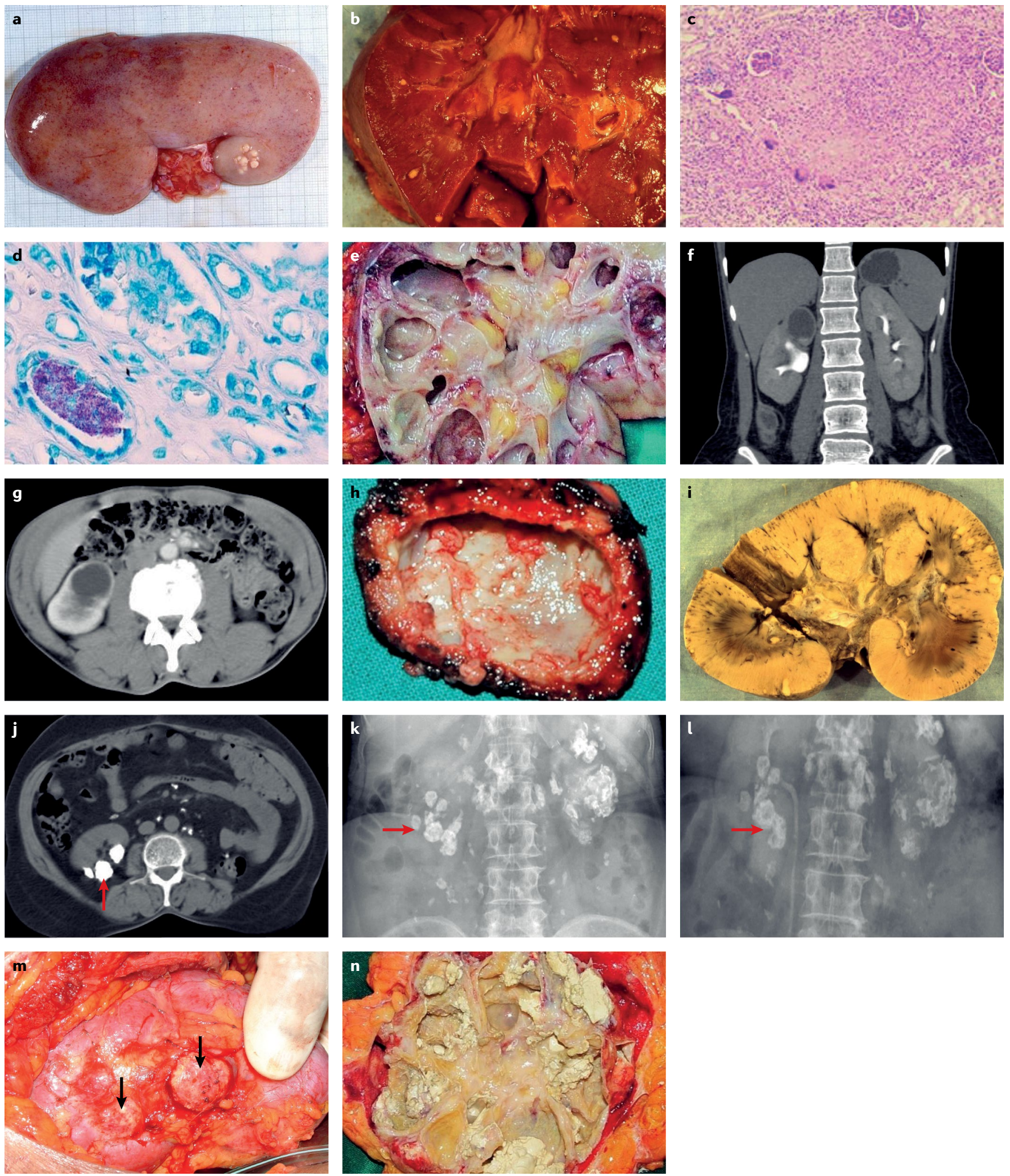

TB of the penis

TB of the penis (FIG. 8) is rare and the literature is limited to isolated case reports. It usually occurs secondary to renal $\mathrm{TB}^{64,17,142-147}$ and following intravesical BCG immunotherapy ${ }^{148}$. In infants, TB of the penis has been reported after ritual circumcision ${ }^{61,84}$.
TB of the urethra

Urethral TB in men and women is rare, despite constant exposure of the urethra to $M t b$-infected urine in renal and ureteric $\mathrm{TB}^{124,15,149}$. Isolated instances of urethral $\mathrm{TB}$ have not been reported. Urethral TB as co-involvement has been described in up to $4.5 \%$ of patients with renal 
4 Fig. 3 | Renal tuberculosis. Autopsy specimens of kidney showing: a | macroscopic caseating lesions on the kidney surface; $\mathbf{b} \mid$ macroscopic caseating lesions in renal parenchyma (bottom left); $\mathbf{c}$ microscopic histological examination showing Mycobacterium tuberculosis (Mtb) granuloma with central caseous necrosis and giant cells; $\mathbf{d} \mid$ numerous acid-fast $M$ tb bacilli (stained red) in renal glomeruli and tubules after Ziehl-Neelsen staining of renal biopsy tissue. e | End-stage renal tuberculosis. Gross pathological specimen of the kidney, depicting extensive destruction of renal tissue and lobar caseation. $\mathbf{f} \mid$ Contrast-enhanced CT image (coronal section) demonstrating non-opacified dilated upper calyx. $\mathbf{g}$ |Contrast-enhanced CT image (horizontal section) demonstrating dilated upper calyx. $\mathbf{h} \mid$ Excised upper polar cavitary lesion with caseous necrosis. i | Pathological specimen showing extensive caseous necrosis and parenchymal destruction ('putty kidney'). j|CT scan (non-enhanced) showing renal parenchymal calcification (arrow). $\mathbf{k}$ | Plain abdominal X-ray depicting classic lobar pattern of calcification (arrow). $\mathbf{l}$ | Intravenous urogram showing non-functioning left kidney and lobar pattern of calcification (arrow). $\mathbf{m}$ | Kidney parenchymal calcifications (arrows) seen during surgery. $\mathbf{n} \mid$ Gross pathological specimen depicting destruction of renal tissue and calcification. Parts a-d and i courtesy of S. Lucas, King's College London.

$\mathrm{TB}^{32,145}$. Acute urethritis with associated prostate $\mathrm{TB}$ or urethral stenosis and fistulae are the common clinical presentations $^{123,124,145,149}$.

\section{TB of female genitalia}

The route of infection of genital TB in women is usually through haematogenous or lymphatic spread of $M t b$ from pulmonary $\mathrm{TB}$, although sexual transmission has been suggested for TB of the cervix, vagina and vulva ${ }^{150-153}$. TB affecting all parts of the female reproductive tract and genitals have been reported ${ }^{26,33,39,40,154-156}$. In India, a country with a high rate of endemic TB, female genital organs affected by TB in order of frequency are: fallopian tubes (95-100\%); uterine endometrium (50-60\%); ovaries (20-30\%); cervix (5-15\%); uterine myometrium (2.5\%); and vagina and/or vulva $(1 \%)^{157}$.

In a clinicopathological study of 1,548 women with genital TB (mean age 29.5 years) ${ }^{158}$, TB of the endometrium was seen in 1,073 patients, TB of the fallopian tubes in 164, TB of the cervix in 157 and 154 had multiple organ involvement. Clinically, 115 women (7.4\%) were diagnosed as having primary infertility and 12 as having secondary infertility; concomitant carcinoma was found in $1.5 \%$ of women ${ }^{158}$.

A pathological study of 1,426 women with genital TB who presented with either primary (94\%) or secondary $(6 \%)$ infertility showed that the fallopian tubes were involved in $100 \%$ of instances, endometrium in $79 \%$, cervix in $24 \%$, vulva and vagina in $0.07 \%$, and ovaries in $11 \%^{26}$. Female genital TB is a chronic disease and can remain subclinical ${ }^{155,156,159}$ with patients not seeking health care for a long time $\mathrm{s}^{33,39,154,155}$. Patients can present with a combination of malaise, abdominal pain, pelvic pain, menstrual irregularity, amenorrhoea, vaginal discharge, postmenopausal bleeding or infertility ${ }^{160-165}$.

UG-TB is closely linked with infertility (FIG. 9) and the pregnancy rates in patients diagnosed with UG-TB are up to $50 \%$ lower than in the general population ${ }^{26,156,166,167}$. TB can remain subclinical and/or is diagnosed late ${ }^{156}$. Genital TB can also cause Asherman syndrome, a triad of oligomenorrhoea, or amenorrhoea with infertility and intrauterine adhesions arising from scar tissue in the uterus and/or the cervix, known as frozen pelvis $^{168}$ (FIG. 9c) . Rarely, genital TB can be associated with the Fitz-Hugh-Curtis syndrome, a disorder that occurs almost exclusively in women characterized by inflammation of the peritoneum and perihepatitis ${ }^{169,170}$. Following TB treatment, the conception rate remains low and, therefore, assisted reproduction techniques are recommended ${ }^{159,167}$.

TB of the cervix, vagina and vulva can present with vaginal bleeding or chronic discharge owing to necrotic ulcerative lesions or tumours ${ }^{152,153,171-176}$. The presentations can be difficult to distinguish clinically from neoplastic or other granulomatous disorders such as cervical amoebiasis, schistosomiasis, brucellosis and sarcoido$\operatorname{sis}^{26,154}$. TB of the vagina and vulva is very rare, presenting as mucosal or skin lesions such as TB verrucosa cutis, erythema induratum or induration of regional inguinal nodes, which can discharge necrotic material (scrofuloderma) $)^{150,151,153,177,178 .}$.

Female genital TB occurs through both haematogenous and lymphatic spread of $M t b$. All of the female reproductive organs are at risk of involvement, although the endometrium and fallopian tubes are most frequently involved. Diagnosis is often delayed and patients often present with primary infertility. Despite treatment, the pregnancy rates following therapy remain low.

\section{Clinical features of UG-TB}

UG-TB can present at any age in men and women, although it is comparatively uncommon in children owing to the long latency period (1-50 years) before reactivating ${ }^{32,100}$. UG-TB can present with a range of clinical manifestations, from asymptomatic, through subclinical, non-specific symptoms and signs, to obstructive uropathy and renal failure ${ }^{12,14-24,32,100,113-119}$. Up to $50 \%$ of cases are diagnosed incidentally when patients are investigated for a range of urinary and genital disorders. UG-TB is often missed clinically because of the lack of awareness among clinicians, its insidious onset, chronic non-specific symptoms, and cryptic and protean clinical manifestations ${ }^{179-181}$. A delay in diagnosis results in disease progression, tissue and organ damage, obstructive uropathy, renal failure and infertility. Health-care workers must have a high clinical awareness of the possibility of underlying $\mathrm{TB}^{179-183}$. This clinical recognition can then lead to a diagnostic work-up with the appropriate investigations and prompt treatment.

\section{Symptoms and signs}

Symptoms, signs and complications of UG-TB are not always defined by the anatomical site of disease $\mathrm{e}^{179-182}$. Many patients can be asymptomatic during the early stages of the disease and have non-localizing symptoms and signs ${ }^{183}$. Autopsy studies ${ }^{34}$ show that only 1 in 5 patients who died had been diagnosed with TB antemortem and that up to $50 \%$ of patients with renal TB were symptomatic ${ }^{32}$. Dysuria, urinary hesitancy and urinary frequency are common findings in renal, bladder and prostatic $\mathrm{TB}$, although patients with renal TB often have associated flank or renal angle pain ${ }^{32,46,179-182}$. Lower urinary tract symptoms are often misdiagnosed as acute bacterial UTIs. In these cases, urinalysis will usually show culture-negative, sterile pyuria and microscopic or macroscopic haematuria ${ }^{12-14,114,115}$. Persistent 
cystitis, haematuria and pyuria should mandate further evaluation, including upper tract imaging, cystoscopy, microbiological investigations, tissue biopsy and histological examination.

Non-specific constitutional symptoms of TB such as fever, weight loss and night sweats are uncommon. If these symptoms are present, they are indicative of concomitant TB outside the urogenital tract, such as pulmonary $\mathrm{TB}^{180-183}$. Some patients can initially present with pulmonary $\mathrm{TB}$ or $\mathrm{TB}$ of other anatomical sites outside the urogenital tract and, therefore, have myriad mixed symptoms. Secondary bacterial infections can occur concomitantly in up to $50 \%$ of patients with UG-TB ${ }^{12-14,180,184}$. Suspicion of UG-TB should arise when conventional antibiotic therapy for suspected UTI is not effective or sterile pyuria is present on examination of urine. Chronic epididymitis or chronic prostatitis that does not resolve with standard antibiotics should also raise a suspicion of $\mathrm{UG}-\mathrm{TB}^{130-135}$.

Renal tuberculosis. Patients with renal TB can remain asymptomatic for many months and the initial presentation might be an incidental abnormal urinaly$\operatorname{sis}^{13,32,46,63,90,185}$. With advancing renal TB, the clinical presentations are non-specific symptoms and signs such as flank pain, dysuria, colic and haematuria and are difficult to distinguish clinically from acute focal bacterial nephritis, focal or global chronic pyelonephritis, or other infectious and non-infectious causes of granulomatous kidney disease $\mathrm{e}^{115,116}$.

Renal TB can lead to chronic renal failure, fistula formation and hypertension ${ }^{46,93,104}$. Other manifestations that are clinically associated with renal TB include rapidly progressive glomerolunephritis ${ }^{186}$, crescentic glomerulonephritis ${ }^{187}$ and membranous nephropathy ${ }^{188}$. Acute and chronic bacterial infections concomitant with
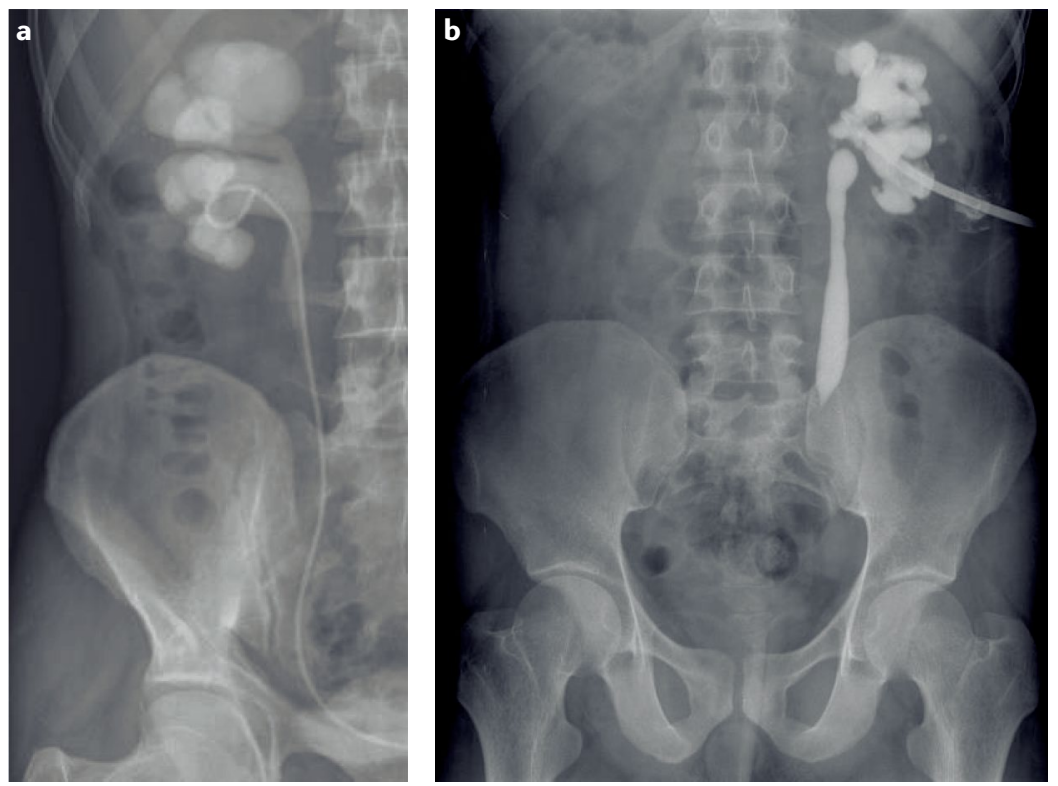

Fig. 4 | Tuberculosis of the ureters. a | Ureteric tuberculosis: excretory urogram showing right mid and distal ureteric stricture with double J stent in situ. $\mathbf{b} \mid$ Renal and ureteric tuberculosis: antegrade nephrostogram depicting long segment mid and distal ureteric strictures.
$\mathrm{TB}$ are common and should arouse suspicion of TB when no response to antibiotic therapy is observed ${ }^{115}$. Chronic TB inflammation of the renal pelvis can also lead to squamous metaplasia ${ }^{91}$, carcinoma ${ }^{189}$ and amyloidosis $^{190}$. Evidence of active TB in the lung might be the only indication of $\mathrm{TB}^{179}$. Rarely, $\mathrm{TB}$ of the renal artery can present with hypertension ${ }^{191}$.

Prostatic tuberculosis. The prostate is the second most frequently reported site of UG-TB ${ }^{32}$. Symptoms and signs of the early stages of prostatic TB are cryptic and many cases remain undiagnosed clinically and are detected incidentally at autopsy ${ }^{32}$, or diagnosed in the laboratory when samples such as expressed prostate secretions, semen and prostate biopsy samples are sent for investigation of cancer or infertility ${ }^{192}$.

Symptoms of prostate TB include acute or chronic pelvic pain caused by prostatitis, dysuria, hesitancy and difficulty in urination, urinary frequency, nocturia, haematospermia, and sexual dysfunction. Urgency is seldom present unless the bladder is affected ${ }^{118,122,134}$. In HIV-infected individuals or those with immunosuppression owing to other causes, patients can present with prostatic abscesses or discharging perineal sinuses ${ }^{193}$.

Digital rectal examination cannot detect early prostatic TB but as the disease progresses, soft areas might be palpable as a result of caseous necrosis ${ }^{33}$. TB of the prostate can be nodular, but the prostate gland itself is non-tender on digital rectal examination ${ }^{118-123}$. The necrotic TB foci are often associated with an elevated serum PSA level, which can be mistaken for prostate cancer $^{118,122,123}$. A micturating cystourethrogram or urethrography can demonstrate dilated prostatic ducts and variable filling associated with destruction of the prostatic tissue ${ }^{123}$. Ultrasonography-guided prostate biopsies of the hyperechoic lesions are required to confirm the diagnosis and exclude an underlying adenocarcinoma ${ }^{194}$.

Ureteral TB. Symptoms of ureteral TB are non-specific and include haematuria, abdominal colic and pain associated with ureteric obstruction ${ }^{12-14,18}$.

Bladder TB. Symptoms of bladder TB are non-specific and include urinary frequency and urgency to micturate, dysuria and haematuria ${ }^{12-18}$.

Scrotal tuberculosis. Scrotal TB (TB epididymo-orchitis) can present with unilateral (66\% of cases) or bilateral (34\% of cases) involvement, acute or chronic, painful, or painless scrotal swellings with scrotal skin inflammation and oedema ${ }^{195,196}$

The presence of a non-tender testicular mass, enlarged, hard and non-tender epididymis, a thickened or beaded vas deferens, or scrotal oedema is suggestive of scrotal $\mathrm{TB}^{197}$. A large distended epididymis occurs secondary to chronic granulomatous tissue and obstruction of the vas deferens. Differentiating these characteristics from malignant swellings can be difficult in the clinic $^{146}$. Granulomatous involvement along the vas deferens gives rise to a beaded appearance ${ }^{140}$. Scrotal fistulae and sinuses discharging thin and odourless pus are suggestive of $\mathrm{TB}^{129,132,133}$. Patients with scrotal TB are 

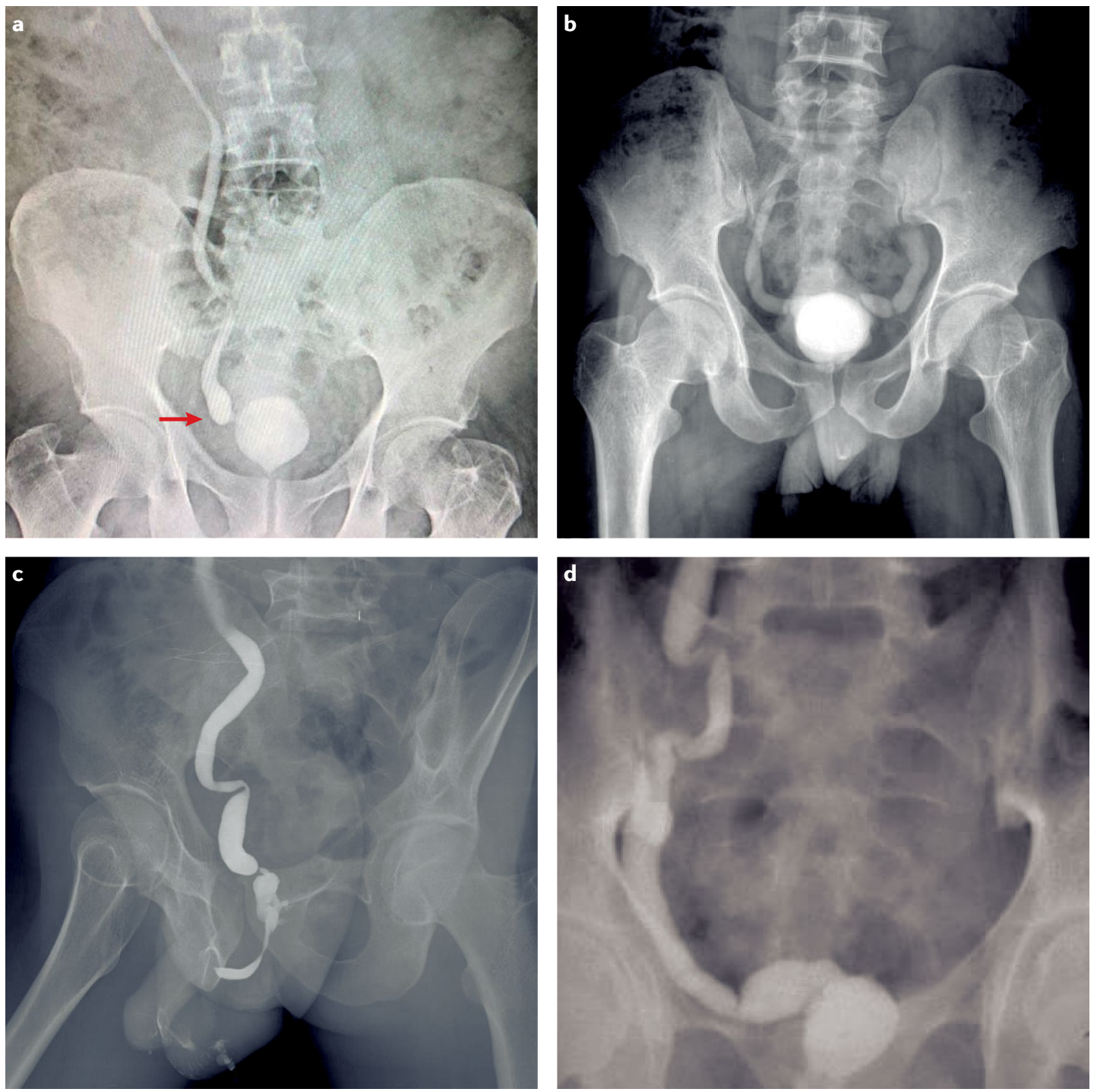

Fig. 5 | Bladder tuberculosis. a | Intravenous urogram image showing right distal ureteric stricture (red arrow) and a small capacity bladder. $\mathbf{b} \mid$ Micturating cystourethrogram showing a small capacity bladder with bilateral vesicoureteral reflux. c|Severely contracted non-redeemable urinary bladder with grade 5 vesicoureteral reflux. $\mathbf{d}$ | Bladder tuberculosis: classical thimble bladder with grade 5 vesicoureteral reflux.

sometimes diagnosed incidentally during investigation for male infertility ${ }^{140,198}$. Patients have oligozoospermia or azoospermia owing to granulomatous destruction and obstruction in the epididymis or vas deferens. TB epididymitis is diagnosed by using fine-needle aspiration cytology or an epididymal biopsy as $M t b$ is not usually present in the urine $e^{129-131}$.

Penile TB. TB of the penis is rare (accounting for $<1 \%$ of UG-TB incidence) and can present with painless or painful single or multiple swellings and ulcers on the penis $^{64,142-147}$. Chronic granulomatous inflammation of the glans penis or cavernosal tissue can manifest as penile skin papules, penile masses, ulcers, nodules or cold abscess with or without inguinal lymphadenopathy. It can be associated with urethritis, urethral discharge, urethral strictures, perineal fistulae or sinuses with a 'watering can' effect' ${ }^{34,142,149}$. TB cavernitis results in chronic penile pain and erectile dysfunction ${ }^{64}$.
Tuberculids are asymptomatic, symmetrical, dusky red papules and pustules over the glans penis, which occur in crops and heal with scarring as a result of acute leukocytoclastic vasculitis and thrombosis of dermal vessels. Ulcerative lesions can mimic penile cancer, genital herpes simplex, granulomatous ulcers of granuloma inguinale, cutaneous leishmaniasis, schistosomiasis and lymphogranuloma venereum ${ }^{117}$. A biopsy should, therefore, always be performed and sent for microbiological, histological and molecular analyses.

Urethral TB. Early symptoms of urethral TB are urethral discomfort and discharge, which are easily missed ${ }^{123,199}$. Acute cases present with urethral discharge and endoscopy can reveal red urethral mucosa that is beefy in appearance and colour with ulceration ${ }^{123,145}$. Chronic urethral infections present with poor urinary flow caused by the development of urethral strictures ${ }^{123,200}$. Any part of the urethra can be involved and present 
clinically with TB of the prostate, prostatic abscess, multiple discharging penile sinus, rectourethral fistulas, or extensive fistulation into the perineum ${ }^{145,149,200}$.

\section{Female genital TB}

Female genital TB has no specific signs or symptoms ${ }^{39}$. Clinical presentation depends on site of involvement and extent of disease $\mathrm{e}^{33,39,155,158,159}$. In the reproductive age group (15-50 years old) in areas with high rates of endemic TB, infertility is the most common presentation, owing to involvement of the ovaries and fallopian tubes $^{40,156,157,159}$. TB of the female reproductive tract can present with primary or secondary infertility. Primary infertility has been reported in many patients with UG-TB ${ }^{167}$. Rarely, female genital TB is associated with the Fitz-Hugh-Curtis syndrome ${ }^{169,170}$. TB of the uterus can cause chronic inflammation and adhesions of the endometrial cavity ${ }^{118,154}$. Patients can present with abdominal masses, tenderness in the suprapubic region and fever - symptoms that are similar to those of pelvic inflammatory disease $\mathrm{e}^{13,39,40}$. When TB of the female urogenital tract presents with pain and an adnexal mass the differential diagnosis is broad and includes acute and chronic bacterial pelvic infections, ectopic pregnancy, endometriosis, cancer (ovarian, uterine, or cervical), and appendicitis ${ }^{18,169}$. Ulcers, masses and granulomatous lesions of the female urogenital tract can be mistaken for cancer or a range of infectious disorders such as syphilis, actinomycosis, granuloma inguinale, lymphogranuloma venereum, elephantiasis (filariasis), Crohn disease, schistosomiasis and amoebiasis ${ }^{26,154}$. Several case reports of TB of the cervix in HIV-infected women have been published ${ }^{80}$. Complications of female UG-TB include vesicovaginal fistula, rectovaginal fistula, tubovesical fistula, urethrocutaneous fistula, tuboperitoneal fistula and tubo-intestinal fistula ${ }^{10,168,169}$.

$T B$ of the ovaries and fallopian tubes. In the early stage of TB of the ovaries and fallopian tubes, patients are asymptomatic ${ }^{13,39,155,156,158,159}$. Many patients are diagnosed incidentally when being investigated for infertility. As the disease progresses, a number of

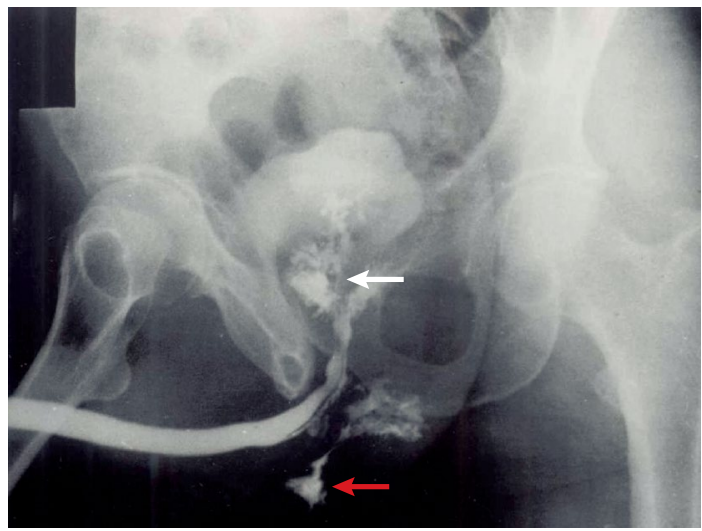

Fig. 6 | Prostate tuberculosis. Contrast fistulogram showing tissue destruction (white arrow) and urethrocutaneous fistula (red arrow). sequelae can occur: chronic inflammation, induration, fibrosis and narrowing of the fallopian tubes and tubo-ovarian abscesses ${ }^{201,202}$, hydrosalpinx, pyosalpinx and tubo-ovarian masses ${ }^{39}$. Ovaries are involved in $20-30 \%$ of women with tubo-ovarian masses, in whom abdominal distension and palpable abdominal lumps and tenderness in the adnexa and suprapubic region can mimic ovarian cancer ${ }^{161,201,203,204}$. Ovarian TB and peritoneal TB can present with acute abdominal pain and mimic appendicitis ${ }^{160,202}$.

TB of the uterus. TB of the uterus is often asymptomatic or presents with non-specific symptoms ${ }^{158,159,205}$. The clinical presentation differs in women of reproductive age (15-50 years) and postmenopausal women ( $\geq 51$ years old). Common symptoms for women of reproductive age are irregular menstrual cycles, abnormal bleeding (menorrhagia, oligomenorrhoea, amenorrhoea, pelvic discomfort, abdominal pain (dysmenorrhea), or primary or secondary infertility). Postmenopausal women present with vaginal bleeding, discharge, pus, pelvic or abdominal pain or swelling ${ }^{10,13,26,39,159,163}$. Presence of fever, loss of weight and anorexia indicate presence of pulmonary TB. Endometrial TB can cause ulcerative, granular or fungating lesions, and the uterine cavity can be distorted, obstructed and destroyed by intrauterine adhesions. Endometrial caseation and ulceration causing intrauterine adhesions are referred to as Asherman syndrome ${ }^{206}$ (FIG. 9c).

TB of the cervix. TB of the cervix is rare ${ }^{171,174,176,207,208}$. Symptoms are non-specific, such as vaginal discharge, bleeding, lower abdominal or pelvic pain, coital discomfort and postcoital bleeding. Examination can reveal papillary or vegetative growths and ulcers that mimic cervical cancer or other granulomatous conditions ${ }^{208}$. Diagnosis can be easily missed. Several cases have been diagnosed incidentally on examination of Pap smears for cervical cancer ${ }^{209}$.

TB of the vagina and vulva. TB of the vagina and vulva is rare ${ }^{150}$. Case reports indicate that it can present with lumps, swelling, hypertrophic lesions, pigmented growths, ulcers, discharging sinuses, elephantiasis and esthiomene, vulval lymphoedema, grossly hypertrophic lesions, or a non-healing ulcer mimicking malignancy that necessitates a biopsy and histopathological examination to confirm the diagnosis ${ }^{150,152-154}$. Rarely, TB of the vagina can cause involvement of the Bartholin glands, as well as vesicovaginal or rectovaginal fistula formation ${ }^{158}$. Labial swellings and ulcerating lesions have been described in HIV-infected individuals $^{210}$. Hypertrophic lesions or swellings caused by TB can be mistaken for vaginal cysts, vulval and vaginal warts, condyloma lata, Bartholin abscess, and vulval or vaginal cancer ${ }^{153,154}$.

\section{Diagnostic work-up}

Early and accurate diagnosis of UG-TB is important for successful treatment outcomes. No single specific diagnostic test exists for UG-TB. Furthermore, detection of $M t b$ is not possible in all cases of TB owing to 

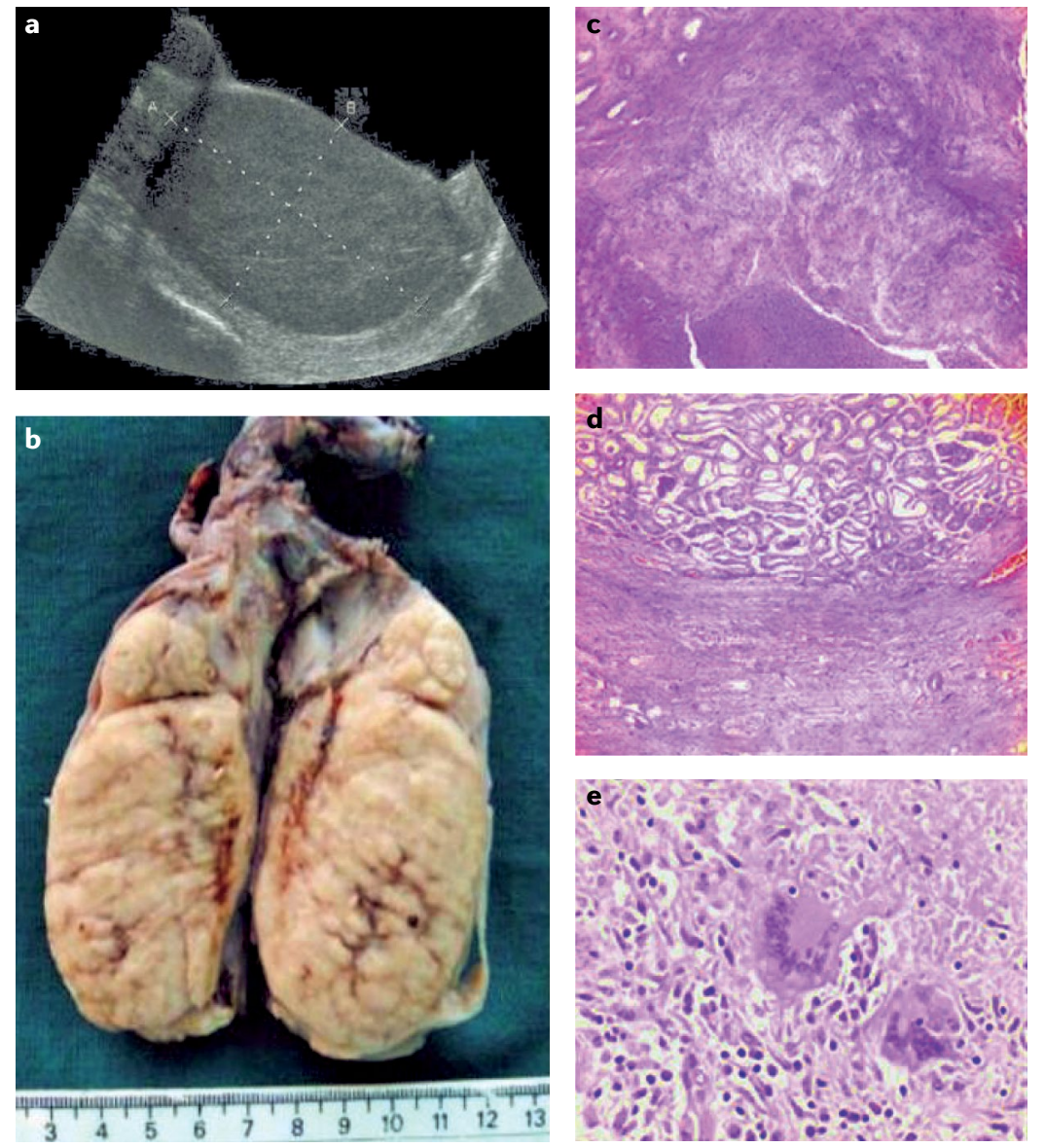

Fig. 7 | Tuberculosis of the testes and seminal vesicles. a | Ultrasonography of testes showing the testicular parenchyma lesions. $\mathbf{b}$ | Gross pathological specimen showing extensive caseating granulomatous tissue replacing testicular tissue. $\mathbf{c} \mid$ Chronic granulomatous inflammation of the testes. $\mathbf{d}$ |Chronic granulomatous inflammation of the seminal vesicles. e| Multinucleated Langhans giant cells within the granulomas. Parts $\mathbf{a}$ and $\mathbf{b}$ adapted from Paul et al., Isolated tuberculous orchitis: a mimicker of testicular malignancy. Indian J. Urol. 26, 284-286 (2010) ${ }^{146}$. Parts c-e courtesy of S. Lucas, King's College London. sent for microscopy should also be sent for culture and molecular analysis.

\section{Smear microscopy}

Smear microscopy refers to microscopic examination of sputum, urine, pus, discharge, prostatic massage fluid, biopsy tissue and sputum for acid-fast bacilli (AFB) performed using Ziehl-Neelsen (ZN) or auramine staining (FIG. 3e). This method has been a first-line diagnostic test for PTB for the past 70 years. Results can be available in $24-48 \mathrm{~h}$ after receipt of samples by the laboratory ${ }^{215}$. Light-emitting diode (LED)-based fluorescent microscopy has similar sensitivities and specificities to light microscopy, but the reading time of LED is three times faster than ZN microscopy $\mathrm{Z}^{2,5,213,216}$. The WHO recommends that conventional fluorescence microscopy be replaced by LED microscopy and, therefore, LED microscopy is being introduced and used as an alternative to conventional ZN light microscopy ${ }^{2,5,213,216}$.

\section{Urine microscopy and culture}

Patients in whom UG-TB is suspected should have three early morning urines on consecutive days sent for AFB microscopy and culture. AFB smear microscopy requires $5 \times 10^{3}$ bacilli $/ \mathrm{ml}$ of specimen to yield a positive result and the sensitivity is low because the numbers of $M t b$ bacilli in urine are small ${ }^{49,218,219}$ and environmental, non-pathogenic mycobacteria such as Mycobacterium smegmatis can contaminate urine and give false-positive results ${ }^{218,220,221}$. A study of 2,240 patients with suspected UG-TB showed that among patients with histologically proven TB, the urine culture yield of $M t b$ was $10.7 \%{ }^{217}$. In patients with renal TB, between 30 and $40 \%$ of single urine specimens will be positive for Mycobacterium culture $^{218}$. When other clinical samples such as bladder and prostate biopsies from the site of urogenital disease are available for microbiological and molecular analyses, the diagnostic yield is much increased ${ }^{219}$.

Over the past 5 years, diagnosis of TB has been revolutionized by moving away from traditional culture using Lowenstein-Jensen medium or liquid culture to $M t b$ culture systems (for example, rapid automated liquid culture systems such as the Becton Dickinson BACTEC MGIT 960), rapid molecular nucleic acid amplification systems line probe assay (LPAs) and the GeneXpert MTB/RIF Assay (Cepheid, Sunnyvale, CA, USA), which provides rapid diagnosis and identifies rifampicin resistance (operationally within $24-48 \mathrm{~h})^{220}$.

Culture-based methods. Culture of clinical specimens for $M t b$ is the gold-standard diagnostic method for the diagnosis of active TB with a sensitivity of $65 \%$ and specificity of $100 \%^{2,5,6,213}$. Traditionally, solid LowensteinJensen culture medium was used, but this method takes 6-8 weeks to detect growth of $M t b$. This method has been phased out in most countries and has been replaced with automated liquid Mycobacteria Growth Indicator Tube (MGIT) culture using the BACTEC MGIT 960 System (Becton Dickinson-BD) ${ }^{213}$, based on modified Middlebrook 7H9 broth, and positive results can be available within $\sim 2$ weeks ${ }^{221}$. This system has a ruthenium pentahydrate oxygen sensor embedded in silicon 


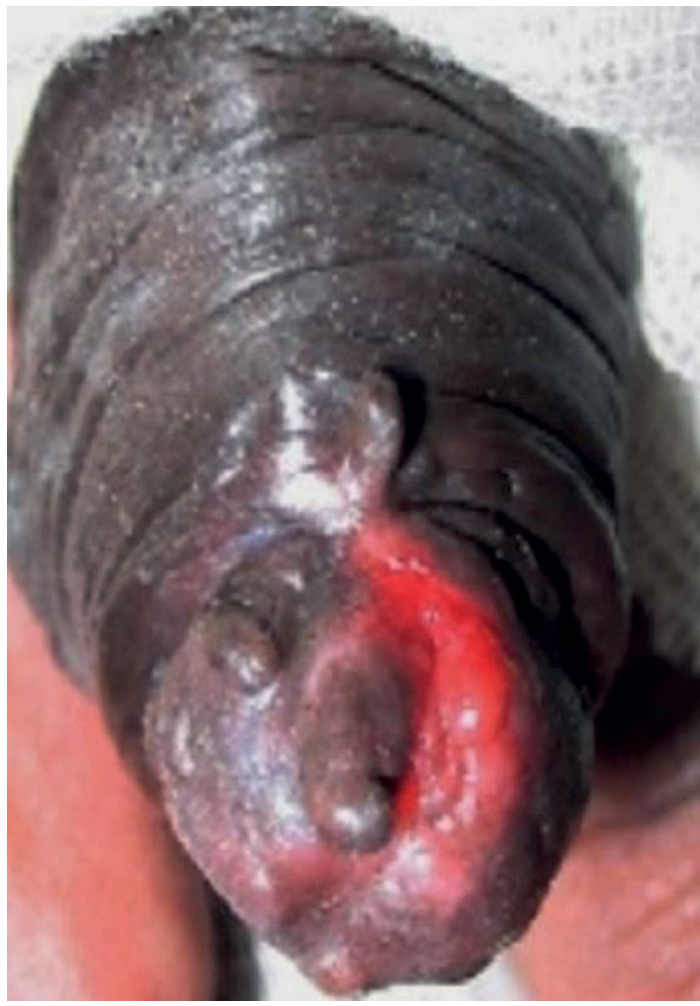

Fig. 8 | Tuberculosis of the penis. Granulomatous ulcerative lesions and nodules on the glans penis. Reprinted from REF. ${ }^{19}$ with permission from the Journal of Clinical and Diagnostic Research.

at the bottom of a tube containing $8 \mathrm{ml}$ of modified Middlebrook 7H9 broth and it fluoresces following the oxygen reduction induced by viable aerobically metabolizing mycobacteria within the medium. The MGIT system scans frequently for increased fluorescence (every $60 \mathrm{~min}$ ), so that any $M t b$ growth can be detected as soon as possible. The MGIT liquid culture system is now recommended by the WHO as the gold-standard confirmatory test for $\mathrm{TB}^{2,5,6,199,214}$. The advantages of liquid culture include its sensitivity, identification of Mycobacterium species and the ability to perform phenotypic drug susceptibility tests (DSTs) and genotyping for further molecular epidemiology studies. The disadvantage of culture methods is the time needed for the growth of mycobacteria. Liquid cultures require at least 9-10 days for positive results and 6 weeks for being considered negative.

\section{The GeneXpert MTB/RIF assay}

After extensive evaluation, the WHO recommended the GeneXpert MTB/RIF assay (Cepheid) as a rapid, affordable near-point-of-care test for detecting $M t b$ and rifampicin resistance simultaneously for patients with pulmonary $\mathrm{TB}^{222}$. This test is a real-time quantitative PCR assay for amplifying $M t b$ DNA and part of the $r p o B$ gene encoding rifampicin resistance ${ }^{212,213,223}$. This assay can give a result in $2 \mathrm{~h}$ and operationally in hospitals and TB clinics within $24 \mathrm{~h}$. The GeneXpert MTB/RIF assay and AFB sputum smear microscopy have the same specificity, but sensitivity of GeneXpert is much higher than AFB smear microscopy using sputum ${ }^{212,213,220}$. The overall sensitivity of a single, direct GeneXpert MTB/ RIF assay test in culture-positive patients was $91 \%$ compared with sensitivity of a single direct microscopy smear test of $59.5 \%$. Mean time to detection is $<1$ day for the GeneXpert MTB/RIF, 1 day for microscopy, 17 days for liquid culture and $>30$ days for solid culture ${ }^{222}$. In HIV-infected individuals, the GeneXpert MTB/RIF assay increases detection of TB by $45 \%$ compared with microscopy and facilitates earlier diagnosis and reduces time-to-initiation of TB treatment ${ }^{212,222}$. The timeliness of detection of rifampicin resistance in adults and children living with HIV using GeneXpert MTB/RIF assay facilitates early initiation of MDR-TB treatment. The GeneXpert MTB/RIF assay is replacing smear microscopy as a first-line TB diagnostic test for detection of pulmonary and extrapulmonary disease worldwide ${ }^{2}$.

\section{Urine-based TB diagnostic tests}

Urine can be readily obtained from patients and is a suitable specimen for detection of $M t b$ using established microbiological methods or with two newer rapid diagnostics tests that have been evaluated: the Xpert MTB/RIF assay, which detects $M t b$ DNA in urine ${ }^{222,224}$ and the lateral flow assay, which detects mycobacterial cell wall glycolipid lipoarabinomannan (LAM).

\section{GeneXpert MTB/RIF assay using urine}

The GeneXpert MTB/RIF assay has been used to diagnose TB by using clinical specimens other than sputum such as urine, pleural fluid, CSF biopsy samples, pus and stool ${ }^{225,226}$. A 2017 study $^{227}$ used urine samples from 37 patients with culture-positive and 44 patients with clinically diagnosed UG-TB and compared the GeneXpert MTB/RIF assay with conventional microbiology. The GeneXpert MTB/RIF assay performed better than microbiology with a sensitivity of $63.0 \%$, compared with microscopy $(18.5 \%, P<0.001)$ and culture $(45.7 \%$, $P=0.027)$. A small incremental diagnostic yield of urine GeneXpert MTB/RIF assay over the urine TB-LAM test has been noted ${ }^{228}$. The role of the GeneXpert MTB/RIF assay for diagnosis of UG-TB using clinical samples other than urine requires further evaluation.

In 2018, the WHO endorsed a new cartridge, Xpert MTB/RIF Ultra, as a replacement for the GeneXpert MTB/RIF assay cartridge as it has increased sensitivity ${ }^{229}$ and the same specificity. The sensitivities of Xpert Ultra compared with Xpert MTB/RIF assay for detection of pulmonary TB are $63 \%$ versus $46 \%$ for smear-negative and culture-positive sputum. Specificities of GeneXpert Ultra and GeneXpert MTB/RIF assays for detection of pulmonary TB were $96 \%$ and $98 \%$, respectively, overall $^{229}$. However, this assay has not yet been evaluated for detection of UG-TB and prospective studies are required. The use of rapid molecular tests is increasing, but microscopy and culture remain necessary for monitoring response to treatment and detecting relapse. $M t b$ DNA lingers in tissues even after treatment and eradication of live mycobacteria and, therefore, gives false-positive test results with GeneXpert MTB/RIF assays ${ }^{213,220}$. 

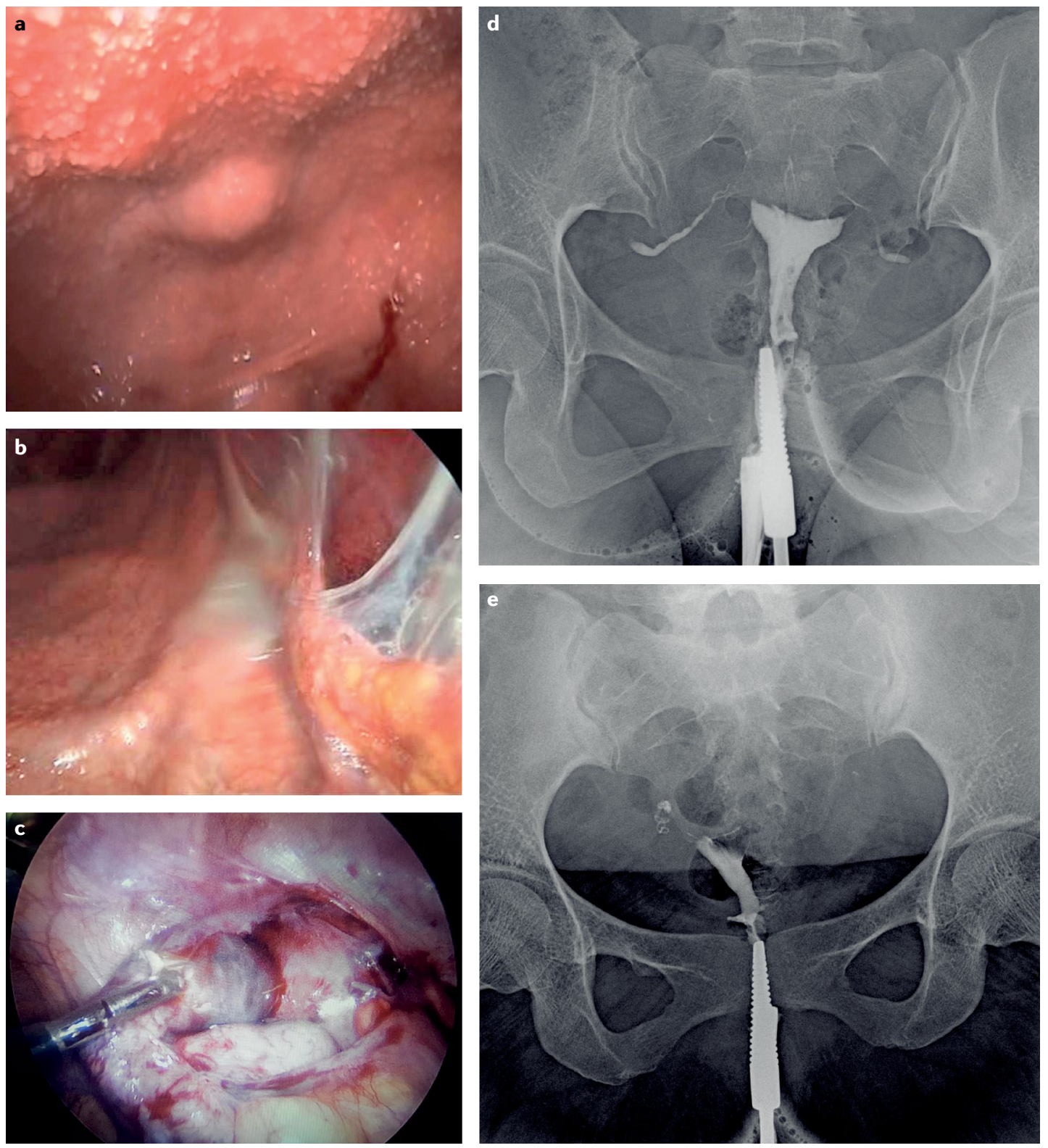

Fig. 9 | Female genital tuberculosis and laparoscopic examination for infertility. a | Miliary tubercles in the Fallopian tube mucosa. b | Pelvic inflammation, dense adhesions and fibrinous exudate. c | Laparoscopic examination shows frozen pelvis (scar tissue and adhesions of the uterus) caused by tuberculosis in a patient with Asherman Syndrome. $\mathbf{d}$ | Hysterosalpingogram investigation for infertility showing bilateral Fallopian tube strictures and blockages. e | Complete blockage of the left Fallopian tube and lack of contrast spillage on the right side indicating distal blockage.

\section{Urine-based LAM assay}

LAM is a constituent part of the cell wall of $M t b$ and can be detected in the urine of patients with active $\mathrm{TB}^{5,6,213,230}$. The lateral flow urine LAM assay (Determine LAM: Alere, Waltham, MA, USA) is an immunochromatographic assay comprising colloidal gold-labelled antibodies attached to LAM, which are captured by immobilized LAM antibodies further along the test strip and form a visual band. The TB-LAM test using urine is currently recommended by the WHO for the diagnosis of HIV-associated TB in people with $\mathrm{CD} 4^{+}$lymphocyte counts $<200$ cells $/ \mu$ l. Patients with advanced immunosuppression are at an increased risk of disseminated $M t b$ infection with consequent renal involvement releasing $M t b$ LAM glycolipid into the urine ${ }^{230}$. The usefulness of this assay for diagnosing UG-TB has not yet been evaluated.

\section{TB drug susceptibility testing}

Ruling out TB caused by drug-resistant $M t b$ strains is important so that specific TB drug therapy can be prescribed. Culture-based phenotypic DST methods are available, but these methods are time-consuming, require sophisticated laboratory infrastructure, qualified staff and strict quality control. The WHO recommends the use of rapid molecular DSTs as the initial tests to detect drug 
resistance before the initiation of appropriate therapy for all $\mathrm{TB}$ patients $\mathrm{s}^{229}$. If rifampicin resistance is detected, further rapid molecular tests for resistance to isoniazid, fluoroquinolones and amikacin should be performed promptly to inform which second-line TB drug therapy should be used for the treatment of rifampicin-resistant TB and MDR-TB. Genotypic DST methods such as next-generation sequencing are attractive alternatives to culture-based DST methods, given the speed of performing molecular methods and the detailed sequencing information that can be generated for multiple gene regions associated with drug resistance ${ }^{229,231}$.

LPAs can detect resistance to rifampicin, isoniazid and other first-line and second-line TB drugs by targeting amplicon regions using membrane-bound probes ${ }^{232}$. LPAs can be used for testing $M t b$ culture isolates (indirect testing), direct testing of specimens that are positive on smear microscopy (first-line LPA (FL-LPA)), and smear-positive and smear-negative sputum specimens (second-line LPA). The FL-LPA has a sensitivity and specificity of $96.7 \%$ and $98.8 \%$, respectively, for detecting resistance to rifampicin and sensitivity and specificity of $90.2 \%$ and $99.2 \%$, respectively, for isoniazid resistance. The WHO has recommended the use of commercially available FL-LPAs (GenoType MTBDRplus V1, GenoType MTBDRplus V2, and Nipro) as initial tests instead of phenotypic DSTs to detect resistance to rifampicin and isoniazid ${ }^{229}$. Other methods for testing resistance to second-line TB drugs are the Hain MTBDRsl assay (Hain Lifescience, Nehren, Germany) (using $M t b$ isolates or smear-positive samples), array-based methods and targeted or next-generation whole-genome sequencing (WGS) ${ }^{213}$. Technologies such as Cepheid Xpert Ultra, Genedrive MTB/RIF (Cepheid) and the chipbased Truenat MTB (Molbio Diagnostics, Goa, India) have been designed for use at peripheral clinics with microscopy facilities to test for rifampicin resistance ${ }^{229}$.

\section{Whole-genome sequencing}

WGS shows promise for further improvements in rapid molecular diagnosis, identification of drug resistance in a range of clinical specimens, and understanding $M t b$ transmission patterns ${ }^{229,231,232}$. Currently, WGS is generally performed only on strains grown in culture owing to the need for a relatively high quantity of good-quality DNA to generate full WGS data for a given sample. WGS can provide the near complete genome of $M t b$ in a sample, whereas targeted next-generation sequencing (NGS) platforms can provide detailed sequence information for multiple gene regions or whole genomes of interest $\mathrm{t}^{232}$. Despite the advantages of NGS over other molecular methods for drug-resistant TB identification and characterization, the uptake of these technologies has been hindered, especially in low-income and middle-income countries, by cost limitations, the need for specialized and well-trained staff, and a lack of readily available data analysis and data storage solutions.

\section{Histological examination}

Histological examination of biological specimens sent from biopsies and fine-needle aspirates ${ }^{233}$ can identify granulomas (FIG. 3c) and acid-fast $M t b$ bacilli (FIG. $3 \mathrm{~d}$ ).
These specimens should also be sent for culture and be processed simultaneously through the GeneXpert MTB/RIF assay and should be cultured to maximize identification of $M t b$ and determine presence of antibiotic-resistant $M t b$ strains. TB can be an incidental histological finding in many patients in whom TB was not considered clinically and biopsy specimens were sent to confirm a clinical diagnosis of cancer ${ }^{143,154,233-235}$.

Granulomatous inflammation in $M t b$-infected tissues seen on histological examination is a hallmark of $\mathrm{TB}^{26,85,97,154}$. The granuloma is a focal compact collection of epithelioid cells, macrophages, lymphocytes, plasma cells, Langhans giant cells, fibroblasts with collagen and a characteristic central caseous necrosis ${ }^{26,85,97,154}$. An acid-fast stain (ZN or Kinyoun acid-fast stain) of the specimen will show the $M t b$ organisms as slender red rods (FIG. 3c). Identification of AFB does not confirm that the organism is $M t b$ and confirmation by culture or molecular methods is required.

In up to one-fifth of patients, TB epididymo-orchitis is only diagnosed after orchidectomy and histological examination of testes ${ }^{234}$. Fine-needle aspiration cytology or an epidydimal biopsy is used to diagnose TB epididymitis, as $M t b$ is not usually present in the urine $^{129,235-237}$. Fine-needle aspiration should be avoided if cancer is suspected ${ }^{236}$ because of the risk of cancer cell spillage. A meta-analysis indicated that scrotal invasion by needle aspiration does not affect systemic recurrence rates or survival if the diagnosis is malignant ${ }^{238}$. If fine-needle aspiration is unsuccessful or if there is no response to drug therapy, the European Association of Urology suggests surgical exploration to obtain tissue for diagnosis ${ }^{134,239}$.

TB is the most common cause of granulomatous interstitial nephritis seen on histological examination ${ }^{116}$. However, the differential diagnosis of granulomatous disease is broad and includes intracellular microorganisms, such as BCG and non-tuberculous mycobacteria, Brucella spp., Treponema spp., Blastomyces spp. and non-infectious granulomatous disorders, such as sarcoidosis and idiopathic vasculitides ${ }^{85}$. To distinguish $M t b$ from other causes of granuloma, biopsy samples should be routinely processed through the GeneXpert MTB/RIF assay for detecting $M t b \mathrm{DNA}^{226,227}$.

Histological examination of biopsies and fine-needle aspirates is an important adjunct to culture and maximizes identification of $M t b$.

\section{Imaging}

The role of imaging investigations in UG-TB is to help localize the site of disease or tissue destruction, assess the extent of involvement, to monitor the effect of treatment and to discover complications. Chest and abdominal X-rays, ultrasonography, IVU, CT, MRI, and positron-emission tomography (PET)-CT are useful in identifying the large range of abnormal features associated with UG-TB (such as abscesses, strictures and fistulae), and enable definition of the extent of reflux, hydroureter and hydronephrosis ${ }^{107,108,239-243}$. Imaging is also useful for targeting biopsy needles to disease sites in order to obtain biopsy tissue or aspirates for histological, microbiological and molecular analyses. Repeating 
imaging over time is also useful for monitoring response to treatment or detecting relapse. A range of abnormal features are associated with UG-TB.

\section{Plain X-ray}

Plain chest and abdominal X-rays are important initial investigations in the diagnostic work-up for UG-TB. As patients with UG-TB can also have concomitant active pulmonary $\mathrm{TB}$, chest $\mathrm{X}$-rays should be performed to detect any lung disease in patients with chronic cough, night sweats, anorexia, weight loss, contact with a person with active TB or a history of previously treated $\mathrm{TB}^{107,244}$. Abdominal or chest X-rays might show abnormal changes caused by active TB disease or healed TB in up to $50 \%$ of patients with UG-TB ${ }^{107}$. Features of active TB on chest X-ray include patchy or lobar consolidation, linear and nodular opacities, cavities (especially in the upper lung lobes), endobronchial thickening, hilar lymph node enlargement, tuberculoma formation and miliary TB. Indications of chronic active TB or healed previous TB are provided by calcifications in the lung, hilar lymph nodes, kidney, spleen, liver and adrenal glands ${ }^{107}$. Renal calcification (nephrocalcinosis) is seen as a granular opacification and is associated with active, granulomatous infection. A dense, punctate calcification is seen in healed tuberculomas ${ }^{107}$. As calcification in UG-TB is common, a range of the differential diagnosis should be considered, such as helminth infections (nematodes, trematodes and cestodes), renal abscesses and aneurysms of the renal artery and others ${ }^{245}$.

\section{Ultrasonography}

In the early stages of UG-TB, ultrasonography might show no changes ${ }^{246}$. The features of renal TB on ultrasonography are similar to acute focal bacterial nephritis or chronic pyelonephritis ${ }^{247}$. Diffuse, infiltrative renal TB has normal appearance on ultrasonography. Granulomas are seen as small, hypoechoic intrarenal masses and are indicative of TB. Mucosal thickening and stenosis of the calyces are detectable as TB advances, and hypoechoic cystic lesions that communicate with the collecting system might be seen ${ }^{247,248}$. The renal parenchyma might show masses with mixed echogenicity, areas of necrosis, caseation, fibrosis and scarring and associated hydronephrosis or renal atrophy. Large TB abscesses or tuberculomas distorting the renal contour can resemble tumours or cysts ${ }^{197}$. Calcification is common in the late stages of the disease and varies from fine punctate calcific foci to calcification of the whole kidney. The differential diagnosis for calcification includes renal schistosomiases, hydatid cysts, renal abscesses and renal artery aneurysms $s^{98,245}$.

Bladder ultrasonography in chronic cases of bladder TB might show a low-capacity bladder with a thick wall ${ }^{107}$ associated with vesico-ureteric reflux ${ }^{249}$. Common findings in prostate TB on transrectal ultrasonography are hypoechoic areas and irregular patterns peripherally ${ }^{249}$. Ultrasonography of scrotal TB can show focal intratesticular areas with diffusely hypoechoic patterns or a testicular mass with lymphadenopathy that is not distinguishable from testicular cancer ${ }^{139}$. Other features of scrotal TB seen on ultrasonography are scrotal wall and tunica albuginea thickening, hydrocele and intratesticular abscesses. In advanced TB, calcification can also occur ${ }^{250}$. The epididymis can appear enlarged, heterogeneous and hypoechoic in the body and tail, associated with hypoechoic testicular lesions or discharging sinuses ${ }^{246,250}$. Abdominal ultrasonography in patients with $\mathrm{TB}$ peritonitis might show wet peritonitis, dry adhesive peritonitis or septated, particulate or loculated ascites. Adnexal tubo-ovarian masses, peritoneal thickening and endometrial involvement are common findings on ultrasonography in women with UG-TB of the genitalia ${ }^{116,251}$.

Ultrasonography is useful for imaging the upper urinary tract in suspected instances of TB. Renal TB might show abscesses, hypoechoic lesions or hydroureteronephrosis owing to strictures developing in the ureter. Scrotal involvement can be associated with hypoechoic lesions and dilatation of the epididymis and vas deferens.

\section{Intravenous urography}

IVU can show a wide variety of radiological findings that are not specific to TB but can help in focusing the clinician on the possibility of TB diagnosis ${ }^{252}$. This investigation provides a simultaneous assessment of urinary tract anatomy and drainage ${ }^{252-255}$ (FIG. 4). It can demonstrate progressive hydronephrosis as the existing strictures might progress as fibrosis occurs during the healing process $^{107}$. Despite being superseded by CT urogram, IVU still has advantages: it gives static and dynamic imaging of the urinary tract and provides anatomical and drainage details of the kidneys and ureters ${ }^{181}$.

In renal $\mathrm{TB}$, the earliest changes seen using IVU include a moth-eaten appearance of the calyces, even before the patient manifests with symptoms of the disease $^{244}$. Other early findings in renal TB include mucosal oedema resulting in irregularity, loss of sharpness of the calyces causing fuzzy or feathery calyx, calyceal erosion and papillary necrosis ${ }^{254,255}$. The ureters are initially dilated or become irregular in appearance when they are involved. Distal ureteric narrowing at the level of the ureterovesical junction is a common finding (FIG. $4 a$, b) in which a standing column of contrast can be seen in the dilated proximal ureter up to the level of obstruction ${ }^{256}$.

Bladder involvement is seen as a small-capacity, thick-walled bladder with an elevated bladder base. The capacity of the urinary bladder might shrink to such a size that it is commonly known as thimble bladder ${ }^{257}$ (FIG. 5d).

The main limitation of IVU is non-visualization of the pelvicalyceal system in obstructive uropathy. In such circumstances, a CT urogram is indicated and can provide anatomical information regarding renal parenchymal cavities, thickened urinary tract walls and hydronephrosis ${ }^{253,254}$.

Although IVU is infrequently used, characteristics can be observed that suggest renal TB. IVU provides anatomical detail as well as showing the drainage from the collecting system.

\section{CT scan and CT urography}

CT scans and CT urography are better than IVU for identifying renal coalesced cortical granulomas, parenchymal masses, scarring and thickened urinary tract 
mucosa ${ }^{258}$. They are indicated in patients suspected of having UG-TB who have normal IVU or ultrasonography. CT scans can show abnormalities associated with TB (such as calcification, dilated calyces, parenchymal loss and extrarenal spread) in greater detail than IVU ${ }^{141,259-261}$. In a study of 53 patients with urinary TB, the most common findings on CT scan were hydroureter, hydrocalyx and hydronephrosis ${ }^{260}$. These occur secondary to fibrosis and stricture formation.

Features of renal TB on CT scan are dependent on stage of disease ${ }^{258}$ and include one or more cysts surrounding a calyx (FIG. 3e), with thinning of the overlying renal cortex, thickened ureteric walls and calyx, hydronephrosis and renal calcification (FIG. $3 f-h$ ). Active inflammation causes focal tissue oedema and focal hypoperfusion owing to vasoconstriction, and these are well-depicted on contrast-enhanced CT scans. Atypical features include low-density nodes in the renal parenchyma, abdominal spleen, liver and lymph node calcification, vertebral destruction and paravertebral abscess. Calcification is present in $>50 \%$ of patients with renal $\mathrm{TB}$, and CT is a sensitive imaging modality to detect $\mathrm{it}^{258}$. Features of ureteral TB on CT scan include multiple strictures of the ureter and ureteropelvic junction, hydronephrosis and hydroureter. In bladder TB, CT scan can show thickening, fibrosis and ulceration along the bladder wall, which can indicate TB cystitis ${ }^{258-260}$. Contrast-enhanced CT scan of the pelvis can visualize caseation, necrosis, sloughing, irregular cavitation of the prostate and calcification ${ }^{141,258}$. These changes are not TB-specific and cannot be distinguished from a pyogenic abscess in the absence of calcifications ${ }^{258,260}$; thus, further investigation is required. CT scan findings include necrotic areas with ring enhancement of abscesses $^{259,260}$.

CT imaging provides more detail than IVU in suspected UG-TB. Cortical masses and granulomas can be detected, as well as calcification throughout the urinary tract. Multidetector CT urography technology (MDCTU) is replacing IVU for the assessment of renal and urinary tract lesions, using reformatted images such as multiplanar reconstruction and maximum intensity projection $^{255}$.

\section{PET-CT imaging}

PET imaging using 2-deoxy-2-[fluorine-18] fluoro-Dglucose $\left({ }^{18} \mathrm{~F}-\mathrm{FDG}\right)$ can provide functional information about sites with active inflammatory and immune cells that use glucose during metabolism ${ }^{262-267}$. Acquiring ${ }^{18}$ F-FDG PET and CT data together combines anatomical and functional information in a single $\operatorname{scan}^{262,263}$; PET and PET-CT imaging are not specific for TB and cannot distinguish TB from cancer and other infectious causes of the abnormality. Preliminary studies of TB in macaques and humans using ${ }^{18} \mathrm{~F}-\mathrm{FDG}$ PET-CT as a research tool indicated that it could have clinical applications as a non-invasive technique for localizing EPTB disease sites that could then be accessed for obtaining tissue biopsy for histology and microbiological examination to diagnose or rule out $\mathrm{TB}^{264,265}$. Distinguishing TB from cancer is challenging, as FDG is taken up by all metabolically active cells ${ }^{26,267}$. Further evaluation of the role of PET-CT in the management of UG-TB is required to assess its role in localizing disease sites to obtain biopsies and monitoring response to treatment.

FDG PET-CT imaging can help identify sites of UG-TB disease, but distinguishing TB from cancer is not possible, limiting its utility for specific diagnosis of UG-TB.

\section{MRI scan}

MRI abnormalities are usually seen in advanced disease $^{128,268,269}$. MRI has been primarily used for the evaluation of kidney TB because of its superior soft-tissue resolution and multiplanar acquisition to $\mathrm{CT}$ scan and ultrasonography. Renal TB parenchymal changes are similar to those of acute pyelonephritis ${ }^{108,268}$. Focal tissue oedema and focal hypoperfusion owing to vasoconstriction is well-depicted on contrast-enhanced MRI. Multiple or solitary renal parenchymal kidney nodules without other urinary tract involvement have been observed ${ }^{268}$ but are rare.

The appearance of prostatic TB on MRI can be separated into multiple nodular and diffuse types ${ }^{270}$. The nodular type is characterized by markedly low signal intensity on T2-weighted images, which is important for the differential diagnosis from prostate cancer ${ }^{268-270}$. Streaky, diffuse and radiating areas of low signal intensity in the prostate (referred to as watermelon skin on T2-weighted MRI) might be specific for TB-induced prostatitis ${ }^{249,270}$. MRI is also useful at showing cavities, sinus tracts, fistulae and extrarenal and extraprostatic spread of $\mathrm{TB}^{128}$. Peripheral enhancement on MRI is seen when abscesses are present in the prostate ${ }^{270}$. Multiparametric MRI is useful for defining the extent of prostatic $\mathrm{TB}$, sinuses or fistulae, peritonitis and abdominal adnexal masses ${ }^{128,269}$.

MRI has a low sensitivity for detecting early lesions of UG-TB and is used primarily for evaluation of renal TB.

\section{Other investigations \\ Endoscopy}

Endoscopic examination (cystourethroscopy, ureteroscopy, hysteroscopy and laparoscopy) can be used for detecting anatomical abnormalities and localization of UG-TB lesions (such as ulcers, growths, masses, cysts, granulomatous tissue, abscesses and fistulas) and to obtain biopsy tissue samples for microbiological, histological and molecular analyses.

In women with UG-TB and women being assessed for infertility, laparoscopy enables visualization of the fallopian tubes, ovaries and peritoneal cavity for tubercle lesions and obtaining biopsies ${ }^{39,205}$. Features of TB include tubercles (FIG. 9a), tubo-ovarian masses, inflamed tubes and ovaries, free murky peritoneal fluid, pelvic adhesions and fibrinous exudates (FIG. 9b), caseating lesions in the rectouterine pouch (Douglas pouch), omental and uterine adhesions (FIG. 9c) and a blue-coloured uterus ${ }^{39,205}$. Hysteroscopy should be combined with laparoscopy to look for endometrial involvement. In men, ureteroscopy can be used to gain access to the prostatic utricle or ejaculatory ducts, visualize the seminal vesicles and obtain a biopsy specimen of abnormal lesions ${ }^{271}$. 


\section{Hysterosalpingography}

Hysterosalpingography is the primary modality used for evaluating uterine, fallopian tube, peritubal and tubal involvement, and tubal patency (FIG. 9d,e) and for identifying any pathological lesions ${ }^{116}$. A range of changes have been seen in women with genital TB affecting the fallopian tubes and uterus ${ }^{40,145,158,168,272,273}$. Most changes are non-specific and related to chronic inflammation and fibrosis, and the characteristics described that are suggestive of TB include obstruction of the fallopian tube, sites of constrictions of the fallopian tube, endometrial adhesions with deformity and obliteration of the endometrial cavity ${ }^{116,254}$. Fallopian tube occlusion at the junction of the ampulla and isthmus and constrictions along the tube (beaded appearance) are common findings ${ }^{116,205}$. Tubal occlusion leads to dilation of the tube, hydrosalpinx and pyosalpinx, which has a golf-club-like appearance. Intraluminal fibrosis and scarring with intraluminal adhesions result in a cobblestone pattern. Multiple granulomatous lesions in the lumen of the tube give a speckled 'leopard spot' appearance when the ampulla is partially filled with radio-opaque contrast ${ }^{274}$.

Hysterosalpingography is primarily used for identifying pathology and evaluating patency of the uterus and fallopian tube and should be used for investigation of infertility and TB.

\section{Blood tests}

Full blood cell count, C-reactive protein (CRP) and renal function tests should be carried out routinely. A raised CRP is a useful laboratory blood biomarker of disease activity and is helpful for assessing the severity of TB and urea, creatinine and estimated GFR are useful for assessing kidney function. Performing these tests regularly can gauge the response to treatment and monitor adverse effects of TB drugs. Haematological and biochemical tests are non-specific and are used as adjuncts to management.

\section{Diagnosis of latent TB infection}

Immunosuppression from any cause, including chronic kidney disease (CKD), dialysis and renal transplantation, can considerably increase the risk of reactivating LTBI $^{51,275-277}$. Those who also become reinfected with $M t b$ are also at high risk of rapidly progressing to developing active disease ${ }^{19}$. Thus, screening for LTBI pretransplant and post-transplant using interferon $-\gamma$ release assays (IGRAs) is important ${ }^{222}$. No gold-standard diagnostic tests are available for the diagnosis of $\mathrm{LTBI}^{5,6}$. Currently, three tests are recommended by the $\mathrm{WHO}^{278}$ for detecting LTBI, the tuberculin skin test (TST) and two IGRAs: QuantiFERON-TB Gold In-Tube and T-SPOT TB. The TST and IGRAs cannot differentiate between LTBI and active disease and they should not be used as diagnostic tests for active TB. In those apparently healthy individuals with a positive IGRA or TST, the decision to treat LBTI using one of several LTBI treatment regimens recommended by $\mathrm{WHO}^{229}$ should not be taken lightly. Before LTBI infection treatment is commenced on the basis of a positive IGRA or TST test, it is crucial that active TB is ruled out by taking a thorough clinical history and doing a physical examination and other relevant investigations. Those with weight loss, night sweats, elevated erythrocyte sedimentation rate or C-reactive protein and abnormal imaging should be investigated further and those found to have active $\mathrm{TB}$, or are strongly suspected of having active TB on a clinical basis, should receive the WHO-recommended quadruple anti-TB drug regimen ${ }^{2,4,278}$, and they should not be given LTBI treatment regimens.

\section{Medical and surgical management}

The primary aims of management of UG-TB are to eradicate $M t b$ infection with TB drug therapy, treat complications and manage comorbidities and risk factors. The diagnosis of UG-TB requires a combination of clinical, pathological and microbiological findings. Every effort should be made to make an accurate diagnosis of TB, but empirical TB treatment on a clinical basis is common in circumstances in which microbiological or molecular tests are negative and other common causes have been ruled out ${ }^{118}$. Patients should receive joint care between TB physicians or respiratory physicians and appropriate specialists if they have other comorbidities such as $\mathrm{HIV}^{279}$. Close follow-up monitoring is required during TB treatment, for tracking adherence, response to therapy, individual TB drug toxic effects, development of TB drug resistance, TB drug levels in renal failure and drug interactions with antiretroviral therapy in HIV-coinfected patients. Up to $50 \%$ of patients with renal failure present with adverse reactions to anti-TB drugs compared with only $26 \%$ of those with normal renal function $^{279}$. Haemodialysis frequently leads to the elimination of most TB drugs; thus, anti-TB drugs should be administered after dialysis ${ }^{279}$.

\section{TB treatment regimens}

The latest WHO recommended treatment guidelines s, $^{2,278-282}$ for drug-sensitive $\mathrm{TB}^{4}$, drug-resistant $\mathrm{TB}^{279}$, $\mathrm{EPTB}^{282}$ and $\mathrm{LTBI}^{278}$ should be followed.

Treatment of drug-sensitive TB. For drug-sensitive TB, a 2-month intensive phase of quadruple therapy with daily first-line TB drugs (such as rifampicin, isoniazid, pyrazinamide and ethambutol), followed by a 4-month continuation phase of two drugs (rifampicin and isoniazid) is prescribed $^{5}$. In immunosuppressed individuals, the continuation phase is extended to 7 months (to ensure eradication of persistent $M t b$ bacilli and prevent relapse) making a total of 9 months of therapy ${ }^{282}$. Only one clinical trial has evaluated a 6-month versus a 9-month intermittent short course of TB therapy in women with genital TB and found no difference in cure and recurrence rates ${ }^{283}$. As with pulmonary $\mathrm{TB}$, this regimen gives a cure rate of $90 \%{ }^{10}$.

Treatment of drug-resistant TB. Drug-resistant TB is now a global problem ${ }^{2,3}$. In 2017, there were 558,000 new cases of drug-resistant TB diagnosed worldwide, of which 490,000 involved MDR-TB ${ }^{281}$. This number is an increase from WHO estimates of the global caseload of MDR-TB from 274,000 in 2000 and 440,000 in 2010 $\left(R_{E F}{ }^{2}\right)$. The number of extensively drug-resistant $\mathrm{TB}$ (XDR-TB, which is defined as resistance to rifampicin, 
isoniazid, quinolones and aminoglycosides) cases reported to WHO was 10,800 , and $8.5 \%$ of patients with MDR-TB in 2017 were estimated have XDR-TB ${ }^{2}$. In cases of MDR-TB and XDR-TB, a longer course of treatment with more toxic drugs is required than is recommended for drug-sensitive $\mathrm{TB}^{281}$. No trial data have been published on treatment of drug-resistant UG-TB, and these investigations are overdue. The WHO recommends a 9-12-month regimen for pulmonary MDR-TB in patients not previously treated with second-line drugs as evidenced by patient history (which is a conditional recommendation as certainty in the evidence is very low $)^{280}$. Intensive 4-6-month phase with gatifloxacin or moxifloxacin, ethionamide or prothionamide, kanamycin or amikacin, high-dose clofazimine or isoniazid, ethambutol, and pyrazinamide is recommended in the WHO guidelines followed by a 5 -month continuation phase of clofazimine, ethambutol, pyrazinamide and gatifloxacin or moxifloxacin ${ }^{280}$. Injectable agents requiring daily, painful, intramuscular administration for several months have been particularly problematic, at times leading to irreversible deafness ${ }^{281}$. Treatment options for MDR-TB are increasingly becoming more individualized as a result of innovations and a new all-oral 20-24-month regimen is being introduced by the $\mathrm{WHO}^{278}$. Under ideal programmatic conditions, MDR-TB cure rates up to $70 \%$ can be achieved ${ }^{276}$. Treatment is started on the basis of the recommended regimens, depending on drug sensitivity patterns of the $M t b$ isolate and toxicities of TB drugs, but individualized treatment regimens are sometimes necessary and care should be delivered by experienced TB physicians. Close follow-up and clinical and laboratory monitoring for toxic effects, development of further resistance and relapse is required ${ }^{87,284,285}$.

Treatment of TB in patients with renal failure and in HIV-infected individuals. British Thoracic Society guidelines ${ }^{279}$ should be followed for patients with CKD, patients not receiving dialysis, patients receiving haemodialysis or peritoneal dialysis, patients on continuous renal replacement therapy, patients who have received a kidney transplant, patients with renal failure and patients co-infected with HIV in lieu of accumulating drug toxicities in renal failure in whom dose adjustments are required and interactions of TB drugs with antiretroviral therapy occur.

Treatment of latent TB infection. WHO guidelines on the management of LTBI should be followed ${ }^{278}$. In 2018, the WHO recommended the following treatment options for LTBI: isoniazid monotherapy for 6 months for treatment of LTBI in both adults and children in countries with high and low TB incidence; rifampicin plus isoniazid daily for 3 months should be offered as an alternative to 6 months of isoniazid monotherapy as preventive treatment for children and adolescents aged $<15$ years in countries with a high TB incidence; and rifapentine and isoniazid weekly for 3 months can be offered as an alternative to 6 months of isoniazid monotherapy as preventive treatment for both adults and children in countries with a high TB incidence. The following options are recommended for treatment of LTBI in countries with a low TB incidence as alternatives to 6 months of isoniazid monotherapy: 9 months of isoniazid; a 3-month regimen of weekly rifapentine plus isoniazid; 3-4 months of isoniazid plus rifampicin; or 3-4 months of rifampicin alone.

\section{Surgical treatment of UG-TB}

TB drug therapy is the mainstay of treatment for active UG-TB disease and surgical treatment as an adjunct to TB drugs is required in up to $50 \%$ of patients during or after TB drug therapy ${ }^{192,286-293}$. Surgery is required for the following clinical settings: drainage for obstructed pelvicalyceal system (internal or external diversion); drainage of abscesses; nephrectomy for non-functioning kidneys; reconstruction of ureters (ureterocalicostomy, reimplantation of ureters and ileal replacement of ureter $)^{294}$; and reconstructive surgery of the bladder to improve the reduction in the functional bladder capacity ${ }^{295}$.

Obstruction to the drainage of urine is one of the common manifestations of UG-TB that warrants surgical intervention. Strictures involving the ureteropelvic junction and distal ureter are more commonly observed than those involving the proximal ureter ${ }^{19}$. With medical treatment, luminal narrowing heals by fibrosis, reinforcing the need for internal stenting with double J stents. Stents keep the lumen patent, act as a splint and prevent further worsening of the stricture. Serial monitoring with IVU or ultrasonography enables an early diagnosis of progression or worsening of hydronephrosis, enabling early surgical intervention in the form of either pyeloplasty (for ureteropelvic junction obstruction) or ureteric reimplantation (for distal ureteric strictures) ${ }^{296}$. Gow et al. ${ }^{297}$ observed that the appearance of calcifications in the renal parenchyma is a sign of an advanced stage of the disease $\mathrm{e}^{297}$. In these settings, healing with medical management alone is often incomplete, further stressing the need for surgical intervention ${ }^{294}$.

Various reconstructive options are available for strictures, including pyeloplasty, ureterocalyceal anastomosis, calyceal reconstruction, uretero-ureteral anastomosis, ureteric reimplantation (for distal ureteric stricture) or ileal interposition (in cases of multiple ureteric long segment ureteric involvement) ${ }^{287-293}$. Reconstructive surgery in UG-TB has major challenges, especially when suture materials do not adhere to an inflamed renal pelvis. Nephrectomies are even more challenging, as dense perinephric adhesions and adjacent organ involvement might be present, making laparoscopic surgeries difficult to perform ${ }^{298}$. Reconstructive surgeries in these patients also have increased intraoperative blood loss, increased conversion to open procedures and prolonged operative time ${ }^{290,299}$. Renal TB can lead to chronic renal failure, necessitating haemodialysis. Patients with bilateral renal failure can sometimes present with acute exacerbation of renal failure. These patients might need an initial diversion followed by definitive repair that can include use of bowel segments such as ileal ureters or augmentation cystoplasty ${ }^{300}$. Bowel interposition might lead to metabolic abnormalities ${ }^{301}$. In patients with pre-existing renal failure, the kidney function could further worsen over time because of the 
absorptive property of the bowel segment. In patients with renal failure requiring a bowel interposition, a short ileal conduit is preferred over an augmentation of the bladder ${ }^{294}$.

Scrotal TB often results in epididymal involvement and obstructive azoospermia ${ }^{302}$. Exploration of the epididymis might reveal dilated tubules suitable for an epididymovasostomy; often, the epididymis is dilated and is associated with chronic pain and requires an epididymectomy ${ }^{303}$. Testicular TB can mimic a germ cell tumour, and the diagnosis is made following radical orchidectomy ${ }^{304}$. Strictures of the ejaculatory ducts can lead to low volume ejaculates ${ }^{198}$; if stricture is limited to the distal part of the duct, then a transurethral resection of the ejaculatory duct can be performed.

\section{Novel diagnostics and therapies}

The priorities for improving management and outcomes of UG-TB include development of new, rapid, easy-to-use TB diagnostic tests for the early and accurate detection of UG-TB; novel therapies for prevention and treatment of complications of UG-TB (such as fibrosis and destructive urogenital tract lesions that arise from chronic granulomatous inflammation and have consequential long-term morbidity and functional disability); effective and improved treatment regimens for MDR-TB; and new more effective vaccines.

For UG-TB and other extrapulmonary sites of TB disease, making a specific TB diagnosis relies on obtaining clinical samples using invasive procedures. Novel biomarkers in breath, blood and urine, which might serve as new TB diagnostics for EPTB (including UG-TB) using easily obtainable clinical samples, are under development ${ }^{213,305-309}$. Cellular and nuclear components of $M t b$ and host inflammatory and immune response products and patterns can be detected directly in blood, sputum or urine of patients with active TB. Urine is a product of filtered blood and contains molecules and products originating from all host organs or components of $M t b$ : for example, the Mycobacterium-specific $30-32-\mathrm{kDa}$ family of three proteins (Ag85A, Ag85B and $\mathrm{Ag} 85 \mathrm{C}$ ) is of interest and the detection of Ag85 in blood and urine is being evaluated ${ }^{308}$. Mtb-specific peptide fragments, such as early secreted antigen -6 and culture filtrate protein-10, can be detected in serum using antibody-labelled and porous discoidal silicon nanoparticles (NanoDisks) and high-throughput mass spectrometry ${ }^{310}$. Most ongoing research studies are based on host-derived biomarkers consisting of transcriptomic, proteomic, metabolomic or cellular markers or combinations of signature biomarkers, which can be applied to easily obtainable samples other than sputum, such as saliva, finger prick blood, urine and expired breath. These biomarkers are being studied to ascertain whether they can, individually or in combination, be correlates of active $\mathrm{TB}$, or correlates of predicting an increased risk of developing active TB.

More effective, less toxic and shorter drug treatment regimens than current therapy, incorporating a combination of new TB drugs, delamanid, bedaquiline, PA-824 (pretomanid) and other repurposed drugs (for example, clofazimine), are under evaluation in clinical trials ${ }^{281}$.
All TB treatment regimens are directed at eliminating $M t b$ and do not address the important issue of tissue and end-organ damage leading to long-term functional disability despite elimination of $M t b$ with anti-TB drugs. Several host-directed therapies (HDTs), such as autologous mesenchymal stromal cell infusion, are under development to use as an adjunct to standard anti-TB drug treatment ${ }^{281,311-315}$. These HDTs are aimed at reducing excessive inflammation, tissue damage and resulting fibrosis to prevent end-organ damage and improve functional treatment outcomes. Studies of autologous mesenchymal stromal cell infusion as adjunct treatment in patients with MDR-TB and XDR-TB are ongoing ${ }^{313}$. These studies are focused on pulmonary $\mathrm{TB}$, but the principles apply to all forms of TB including UG-TB. In animal models of bladder TB in New Zealand rabbits, a single interstitial injection of autologous bone marrow-derived mesenchymal stem cells (MSCs) showed considerable reduction of inflammation, reduction in development of fibrosis and bladder wall deformity ${ }^{314}$. Translation into human studies of autologous MSC infusion for reduction of fibrosis and scarring of the urogenital tract is required.

\section{Prevention}

Health-care workers who provide care for patients with UG-TB must follow infection control procedures to ensure that $M t b$ infection is not passed from one person to another. No clear guidelines or evidence base exists for reducing the risk of spread to surgical staff from patients with UG-TB who need surgery. Initiating anti-TB treatment at least 8 weeks before surgical intervention takes place is prudent to reduce $M t b$ bacillary load $^{314}$. Standard infection control procedures apply for nursing in patients with UG-TB.

The only available vaccine for TB prevention is the BCG vaccine, which has existed for 80 years and is routinely used in neonates and infants in developing countries $^{316}$. BCG vaccine has a documented protective effect against meningitis and disseminated TB in children. It does not prevent primary infection and, more importantly, does not prevent reactivation of latent pulmonary infection. The past two decades have seen increased efforts at developing new pre-exposure and postexposure vaccines and currently 15 new candidate vaccines are under investigation ${ }^{316}$. Hopes for accelerated vaccine development and evaluation come from renewed political and funder investments that were committed to at the United Nations General Assembly High Level Meeting on TB on 28 September 2018 (UNG-HLM-TB) ${ }^{317}$.

\section{Conclusions}

$\mathrm{TB}$ is a curable and preventable disease, but it remains the leading infectious disease cause of death worldwide. Between $15 \%$ and $40 \%$ of the global burden of the 10 million annual cases of TB present with EPTB. UG-TB is the third most common presentation of EPTB after lymph node TB and pleural TB; however, UG-TB remains a neglected clinical issue. Delays in making a diagnosis result in disease progression, tissue and end-organ damage, and renal failure. UG-TB can present with chronic urinary tract inflammation, haematuria, sterile pyuria, 
abnormal renal function tests, obstructive uropathy, infertility, or renal or testicular mass and can contribute to the development of urothelial cancer and amyloidosis. UG-TB is often missed clinically owing to its insidious onset, chronic non-specific symptoms and cryptic and protean clinical manifestations. Being an elusive clinical condition, the delay in making a diagnosis results in disease progression, ureteral strictures, contracted bladder, obstructive nephropathy, renal parenchymal destruction, irreversible organ damage and end-stage renal failure. Increased clinical awareness and early diagnosis of UG-TB is required. Treatment of UG-TB should follow WHO-recommended treatment guidelines and supplemented by surgery whenever indicated.

Published online 23 September 2019
1. WHO. The top 10 causes of death. WHO https://www. who.int/news-room/fact-sheets/detail/the-top10-causes-of-death (2019).

2. WHO. WHO Global Tuberculosis report 2018. WHO http://who.int/tb/publications/global_report/en/ (2018). 3. Floyd, K., Glaziou, P., Zumla, A. \& Raviglione, M. The global tuberculosis epidemic and progress in care, prevention, and research: an overview in year 3 of the End TB era. Lancet Respir. Med. 6, 299-314 (2018).

4. WHO. Guidelines for the treatment of drug-susceptible tuberculosis and patient care. 2017 update. WHO http://apps.who.int/iris/bitstream/10665/255052/1/ 9789241550000-eng.pdf?ua=1 (2017)

5. Lawn, S. D. \& Zumla, A. I. Tuberculosis (Seminar). Lancet 378, 57-72 (2011).

6. Furin, J., Cox, H. \& Pai, M. Tuberculosis. Lancet 393 , 1642-1656 (2019).

7. Kulchavenya, E. Extrapulmonary tuberculosis: are statistical reports accurate? Ther. Adv. Infect. Dis. 2, 61-70 (2014).

8. Porter, M. F. III Uro-genital tuberculosis in the male. Ann. Surg. 20, 396-405 (1894).

9. Wildbolz, H. Ueber urogenical tuberkulose. Schweiz. Med. Wochenschr. 67, 1125 (1937).

10. Kulchavenya, E., Naber, K. \& Bjerklund Johansen, T. E. Urogenital tuberculosis: classification, diagnosis, and treatment. Eur. Urol. 15, 112-121 (2016).

11. Adhikari, S. \& Basnyat, B. Extrapulmonary tuberculosis: a debilitating and often neglected public health problem. BMJ Case Rep. 11, e226098 (2018).

12. Abbara, A. \& Davidson, R. N. Etiology and management of genitourinary tuberculosis. Nat. Rev. Urol. 8, 678-688 (2011).

13. Figueiredo, A. A. \& Lucon, A. Urogenital tuberculosis: update and review of 8961 cases from the world literature. Rev. Urol. 10, 207-217 (2008).

14. Gow, J. \& Barbosa, S. Genitourinary tuberculosis: a study of 1117 cases over a period of 34 years. Br. J. Urol. 56, 449-455 (1984).

15. Ishibashi, Y., Takeda, T., Nishimura, R. \& Ohshima, H. A clinical observation of genitourinary tract tuberculosis during the last decade. HinyokikaKiy 31, 107-112 (1985)

16. Chtourou, M. et al. Management of genito-urinary tuberculosis. A report of 225 cases. J. Urol. 161, 9 (1999).

17. Gokce, G. et al. Genitourinary tuberculosis: review of 174 cases. Scand. J. Infect. Dis. 34, 338-340 (2002)

18. Ferrie, B. G. \& Rundle, J. S. H. Genito-urinary tuberculosis in Glasgow 1970 to 1979: a review of 230 patients. Scott. Med. J. 30, 30-34 (1985).

19. Krishnamoorthy, S. et al. Aspects of evolving genito urinary tuberculosis - a profile of genito urinary tuberculosis (GUTB) in 110 patients. J. Clin. Diagn. Res. 11, PC01-PC05 (2017).

20. Christensen, W. I. Genitourinary tuberculosis. Review of 102 cases. Medicine 53, 377-390 (1974)

21. Soriano-Rosas, J. et al. AIDS-associated nephropathy: 5-year retrospective morphologic analysis of 87 cases. Pathol. Res. Pract. 194, 567-570 (1998).

22. Hsieh, H. C. et al. Uro-genital tuberculosis in a medical center in southern Taiwan: an eleven-year experience. J. Microbiol. Immunol. Infect. 39, 408-413 (2006).

23. Lessnau, K. D. et al. Tuberculosis of the genito-urinary system overview of GUTB. Medscape http://emedicine. medscape.com/article/450651-overview\#aw2aab6b4. (2015).

24. Ye, Y. et al. Clinical features and drug-resistance profile of urinary tuberculosis in south-western China: a cross-sectional study. Medicine 95, e3537 (2016).

25. Garcia-Rodrīguez, J. Ä., García Sanchez, J. E. \& Muñoz Bellido, J. L. Genitourinary tuberculosis in Spain: review of 81 cases. Clin. Infect. Dis. 18 557-561 (1994).

26. Nogales-Ortiz, F., Tarancón, I. \& Nogales, F. F. Jr. The pathology of female genital tuberculosis. A 31 -year study of 1436 cases. Obstet. Gynecol. 53, 422 (1979).
27. Grange, J. M. in Tuberculosis - A Comprehensive Clinical Reference. 44-59 (eds Schaff, S. \& Alimuddin Zumla, A.) (Saunders Elsevier, 2009).

28. Riojas, M. A., McGough, K. J., Rider-Riojas, C. J., Rastogi, N. ¿ Hazbón, M. H. Phylogenomic analysis of the species of the Mycobacterium tuberculosis complex demonstrates that Mycobacterium africanum, Mycobacterium bovis, Mycobacterium caprae, Mycobacterium microti and Mycobacterium pinnipedii are later heterotypic synonyms of Mycobacterium tuberculosis. Int. J. Syst. Evol. Microbiol. 68, 324-332 (2018).

29. Watt, C. J., Hosseini, S. M., Lonnroth, K., Williams, B. C. \& Dye, C. in Tuberculosis - a comprehensive clinical reference. 37 (eds Schaff, S. \& Alimuddin Zumla, A.) (Saunders Elsevier, 2009).

30. Silva, M. R. et al. Risk factors for human Mycobacterium bovis infections in an urban area of Brazil. Mem. Inst. Oswaldo Cruz 113, e170445 (2018).

31. Zachoval, R. et al. The incidence of subclinical forms of urogenital tuberculosis in patients with pulmonary tuberculosis. J. Infect. Publ. Health 11, 243-245 (2018).

32. Figueiredo, A. A., Lucon, A. M. \& Junior, R. F. Epidemiology of urogenital tuberculosis worldwide. Int. J. Urol. 15, 827-832 (2008).

33. Yadav, S., Singh, P., Hemal, A. \& Kumar, R. Genital tuberculosis: current status of diagnosis and management. Transl. Androl. Urol. 6, 222-233 (2017).

34. Schubert, G. E., Haltaufderheide, T. \& Golz, R. Frequency of urogenital tuberculosis in an unselected autopsy series from 1928 to 1949 and 1976 to 1989. Eur. Urol. 21, 216-223 (1992).

35. Medlar, E., Spain, D. \& Holliday, R. Post mortem compared with clinical diagnosis of genitourinary tuberculosis in adult males. J. Urol. 61, 1078-1088 (1949).

36. Vithalani, N. \& Udani, P. M. A study of 292 autopsies proved cases of tuberculosis. Indian J. Tuber. $\mathbf{2 9}$, 93-97 (1982)

37. Lanjewar, D. N., Ansari, M. A., Shetty, C. R., Maheshwary, M. B. \& Jain, P. Renal lesions associated with AIDS - an autopsy study. Indian J. Pathol. Microbiol. 42, 63-68 (1999).

38. Perez, S., Andrade, M., Bergel, P., Bracho, Y. \& de Waard, J. H. A simple algorithm for the diagnosis of AIDS-associated genitourinary tuberculosis. Clin. Infect. Dis. 42, 1807-1808 (2006).

39. Grace, G. A., Devaleenal, D. B. \& Natrajan, M. Genital tuberculosis in females. Indian J. Med. Res. 145, 425-436 (2017)

40. Parvez, R. et al. Prevalence of female genital tuberculosis, its risk factors and associated clinical features among the women of Andaman Islands, India: a community-based study. Publ. Health 148, 56-62 (2017).

41. Naing, C., Mak, J. W., Maung, M., Wong, S. F. \& Kassim, A. I. Meta-analysis: the association between HIV infection and extrapulmonary tuberculosis. Lung 191, 27-34 (2013)

42. Fanosie, A. et al. Mycobacterium tuberculosis complex and HIV co-infection among xxtrapulmonary tuberculosis suspected cases at the University of Gondar Hospital, Northwestern Ethiopia. PLOS ONE 11, e0150646 (2016).

43. Tubach, F. et al. Risk of tuberculosis is higher with anti-tumor necrosis factor monoclonal antibody therapy than with soluble tumor necrosis factor receptor therapthehe three-year prospective French Research Axed on Tolerance of Biotherapies registry. Arthritis Rheum. 60, 1884-1894 (2009).

44. Ferrara, G. et al. Risk factors associated with pulmonary tuberculosis: smoking, diabetes and anti-TNFa drugs. Curr. Opin. Pulm. Med. 18, 233-240 (2012).

45. Chattopadhyay, A. et al. Genitourinary tuberculosis in pediatric surgical practice. J. Pediatr. Surg. 32 , 1283-1286 (1997).
46. Narayana, A. S. Overview of renal tuberculosis. Urology 19, 231-237 (1982)

47. Rutkowski, B., Gillow, A. S., Kustosz, J., Liberek, T. $\&$ Zdrojewski, Z. Increasing incidence of tuberculosis in hemodialysis patients. Dial. Transplant. 26, 21-25 (1997).

48. Ulubay, G. et al. 10-year experience of tuberculosis in solid-organ transplant recipients. Exp. Clin. Transplant. 13, 214-218 (2015)

49. Gras, J. et al. Rapid diagnosis of tuberculosis through the detection of mycobacterial DNA in urine by nucleic acid amplification methods. Lancet Infect. Dis. 9 505-511 (2009).

50. Higuita, L. M. et al. Tuberculosis in renal transplant patients: the experience of a single center in Medellín-Colombia, 2005-2013. J. Bras. Nefrol. 36 512-518 (2014).

51. Reis-Santos, B., Gomes, T., Horta, B. L. \& Maciel, E. L. Tuberculosis prevalence in renal transplant recipients: systematic review and meta-analysis. J. Bras. Nefrol. 35, 206-213 (2013).

52. Anand, M., Nayyar, E., Concepcion, B., Salani, M $\&$ Schaefer, $\mathrm{H}$. Tuberculosis in kidney transplant recipients: a case series. World J. Transplant. 7 213-221 (2017)

53. Dharmadhikari, A. S. \& Nardell, E. A. in Tuberculosis - A Comprehensive Clinical Reference 8-16 (eds Schaff, S. \& Alimuddin Zumla, A.) (Saunders Elsevier, 2009).

54. Chang, C. W. et al. Congenital tuberculosis: case report and review of the literature. Paediatr. Int. Child Health. 19, 1-4 (2017).

55. Dewan, P., Gomber, S. \& Das, S. Congenital tuberculosis: a rare manifestation of a common diseases. Paediatr. Int. Child Health 34, 60-62 (2014).

56. Newberry, D. M. \& Robertson-Bell, T. Congenital tuberculosis: a new concern in neonatal intensive care units. Adv. Neonatal Care 18, 341-349 (2018).

57. Lhadon, T. \& Jullien, S. Congenital multidrug-resistant tuberculosis in a neonate: a case report. J. Trop. Pediatr. 65, 188-191 (2018).

58. Aldana-Aguirre, J C El-Hakim, H., Phillipos, E. \& Landry, M. A. Congenital tuberculosis presenting as otorrhoea in a preterm infant. BMJ Case Rep. 2018, bcr-2017-221797 (2018).

59. Fang, X., Mai, R., Guo, J. \& Lin, N. A pre-term infant of 32 weeks gestation with congenital tuberculosis treated successfully with antituberculosis chemotherapy. Paediatr. Int. Child Health 14, 1-3 (2017).

60. Raj, P. \& Sarin, Y. K. Congential tuberculosis in a neonate: a diagnostic dilemma. J. Neonatal Surg. 3 49 (2014).

61. Angus, B. J., Yates, M., Conlon, C. \& Byren, I. Cutaneous tuberculosis of the penis and sexual transmission of tuberculosis confirmed by molecular typing. Clin. Infect. Dis. 33, E132-E134 (2001).

62. Veenema, R. J. \& Lattimer, J. K. Genital tuberculosis in the male: clinical pathology and effect on fertility. J. Urol. 78, 65-77 (1957).

63. Lattimer, J. Renal tuberculosis. N. Engl. J. Med. 273 208-211 (1965)

64. Venyo, A. K. Tuberculosis of the penis: a review of the literature. Scientifica 2015, 601-624 (2015).

65. Hesseling, A. C. et al. Consensus statement on the revised World Health Organization recommendations for BCG vaccination in HIV-infected infants. Int. J. Tuberc. Lung Dis. 12, 1376-1379 (2008)

66. Von Reyn, C. F. et al. Disseminated tuberculosis in human immunodeficiency virus infection: ineffective immunity, polyclonal disease and high mortality. Int. J. Tuberc. Lung Dis. 8, 1087-1089 (2011).

67. Sihra, N., Diasuke, N., Thurairaja, R., Khan, M. S $\&$ Malde, S. Renal tuberculosis following intravesical bacillus Calmette-Guérin for high-grade non-muscleinvasive bladder cancer. Urology 107, e3-e4 (2017). 
68. Lamm, D. Efficacy and safety of bacille Calmette Guerin immunotherapy in superficial bladder cancer. Clin. Infect. Dis. 31, 86-90 (2000).

69. Falkensammer, C. et al. Late occurrence of bilateral tuberculous-like epididymo-orchitis after intravesical bacille Calmette-Guerin therapy for superficial bladder carcinoma. Urology 65, 175 (2005).

70. Colomba, C. et al. Case of epididymo-orchitis after intravesical bacille Calmette-Guérin therapy for superficial bladder carcinoma in a patient with latent tuberculosis infection. Infect. Agent. Cancer 11, 25 (2016).

71. Lee, S. Y. \& Choi, S. H. Treatment experience for incidentally diagnosed asymptomatic prostate tuberculosis in a patient with history of BCG intravesical therapy. Urol. Case Rep. 17, 39-41 (2017).

72. Klebanov, N. \& Raghavan, A. Tuberculous orchitis following intravesical Bacille Calmette-Guérin (BCG) therapy. Cureus 10, e2703 (2018).

73. Hunter, R. L. Tuberculosis as a three-act play: A new paradigm for the pathogenesis of pulmonary tuberculosis. Tuberculosis 97, 8-17 (2016).

74. Rao, M. et al. Latent TB Infection (LTBI) Mycobacterium tuberculosis pathogenesis and the dynamics of the granuloma battleground. Int. J. Infect. Dis. 80S, S58-S61 (2019)

75. Bezuidenbhout, J. \& Schneider, J. W. in Tuberculosis - A Comprehensive Clinical Reference 117-128 (eds Schaff, S. \& Alimuddin Zumla, A.) (Saunders Elsevier, 2009)

76. Menzies, N. A. et al. Progression from latent infection to active disease in dynamic tuberculosis transmission models: a systematic review of the validity of modelling assumptions. Lancet Infect. Dis. 18, e228-e238 (2018)

77. Salvatore, P. P., Proaño, A., Kendall, E. A., Gilman, R. H. \& Dowdy, D. W. Linking Individual natural history to population outcomes in tuberculosis. J. Infect. Dis. 217, 112-121 (2017)

78. Vynnycky, E. \& Fine, P. E. The natural history of tuberculosis: the implications of age-dependent risks of disease and the role of reinfection. Epidemiol. Infect. 119, 183-201 (1997).

79. Simmons, J. D. et al. Immunological mechanisms of human resistance to persistent Mycobacterium tuberculosis infection. Nat. Rev. Immunol. 18 575-589 (2018)

80. Lamba, H., Byrne, M., Goldin, R. \& Jenkins, C. Tuberculosis of the cervix: case presentation and a review of the literature. Sex. Transm. Infect. 78, 62-63 (2002).

81. Aphonin, A. B. Perezmanas, E. O., Toporkova, E. E. $§$ Khodakovsky, E. P. Tuberculous infection as sexually transmitted infection. Vestn. Poslediplomnogo Obrazovaniya 3, 69-71 (2006).

82. Regmi, S. K., Singh, U. B., Sharma, J. B. \& Kumar, R. Relevance of semen polymerase chain reaction positive for tuberculosis in asymptomatic men undergoing infertility evaluation. J. Hum. Reprod. Sci. 8, 165-169 (2015)

83. Barmon, D., Kataki, A. C., Sharma, J. D. \& Gharpholia, D. A case of cervical tuberculosis mimicking cervical carcinoma. J. Obstet. Gynaecol. India. 63, 285-287 (2013).

84. Holt, L. E. Tuberculosis acquired through ritual circumcision. J. Am. Med. Associ. 61, 99-102 (1913).

85. Zumla, A. \& James, D. G. Granulomatous infections: etiology and classification. Clin. Infect. Dis. $\mathbf{2 3}$, 146-158 (1996)

86. Houben, R. M. \& Dodd, P. J. The global burden of latent tuberculosis infection: a re-estimation using mathematical modelling. PLOS Med. 13, e1002152 (2016)

87. Butler, M. R. \& O'Flynn, D. Reactivation of genitourinary tuberculosis. Eur. Urol. 1, 14-17 (1975).

88. Arora, N., Saha, A. \& Kaur, M. Tuberculous pyelonephritis in children: three case reports. Paediatr. Int. Child Health. 37, 292-297 (2017).

89. Kretschmer, H. L. Tuberculosis of the kidney, a critical review based on a series of 221 cases. N. Engl. J. Med. 202, 660-671 (1930).

90. Ross, J. C. Renal tuberculosis. Br. J. Urol. 25 277-292 (1953)

91. Eastwood, J. B., Corbishley, C. M. \& Grange, J. M. Tuberculosis and the kidney. J. Am. Soc. Nephrol. 12, 1307-1314 (2001)

92. Qiu, S. P. et al. A clinical study of 281 cases of renal tuberculosis. Chin. J. Urol. 23, 398-400 (2002).

93. Daher, E. F., Bezerra da Silva, G. \& Guardao Barros, E. J. Renal tuberculosis in the modern era. Am. Trop. Med. Hyg. 88, 54-64 (2013).
94. Medlar, E. M. Cases of renal infection in pulmonary tuberculosis: evidence of healed tuberculous lesions. Am. J. Pathol. 2, 401-411 (1926).

95. Medlar, E. M. \& Sasano, K. T. Experimental renal tuberculosis, with special reference to excretory bacilluria. Am. Rev. Tuberc. 10, 370-377 (1924).

96. Marques, L. P., Rioja, L. S., Oliveira, C. A. \& Santos, O. D. AIDS-associated renal tuberculosis. Nephron 74, 701-704 (1996).

97. Shah, S., Carter-Monroe, N. \& Atta, M. G. Granulomatous interstitial nephritis. Clin. Kidney J. 5 516-523 (2015)

98. Prakash, J., Goel, A. \& Sankhwar, Singh, B. Extensive renal and ureteral calcification due to tuberculosis: rare images for an uncommon condition. BMJ Case Rep. 2013, bcr2012008508 (2013).

99. Patil, S. B., Desai, A. S., Biradar, A. N. \& Kundargi, V. S. Extensive nephroureteric calcification presenting with renal failure: a rare case report. Urol. Ann. 7 375-377 (2015)

100. Kulchavenya, E. V., Shevchenko, S. Y. \& Cherednichenko, A. G. Diagnosis and treatment of cystitis: more questions than answers? Urologiia 5 , 37-42 (2016)

101. Lima, N. A. et al. Review of uro-genital tuberculosis with focus on end-stage renal disease. Rev. Inst. Med. Trop. Sao Paulo 54, 57-60 (2012).

102. Mallinson, W. J., Fuller, R. W., Levoson, D. A Baker, L. R. \& Cattel, W. R. Diffuse interstitial renal tuberculosis an unusual cause of renal failure. $Q$. J. Med 50, 137-148 (1981)

103. Eastwood, J. B. Zaidi, M., Maxwell, J. D., Wing, A. J. $\&$ Pazianas, M. Tuberculosis as primary renal diagnosis in end-stage uraemia. J. Nephrol. 7, 290-293 (1994)

104. Oliverira, J. L., Silva Junior, G. B. \& Daher, E. F. Tuberculosis associated chronic kidney disease. Am. J. Trop. Med. Hyg. 84, 843-844 (2011).

105. Lee, K. S. et al. Laparoscopic nephrectomy for tuberculous non-functioning kidney: comparison with laparoscopic simple nephrectomy for other diseases. Urology 60, 411-414 (2002).

106. Hemal, A. K., Gupta, N. P. \& Rajeev, K Comparison of retroperitoneoscopic nephrectomy with open surgery for tuberculous non-functioning kidneys. J. Urol. 164, 32-35 (2000)

107. Merchant, S., Bharati, A. \& Merchant, N. Tuberculosis of the uro-genital system - urinary tract tuberculosis: renal tuberculosis. I. Indian J. Radiol. Imaging 23 46-63 (2013)

108. Merchant, S., Bharati, A. \& Merchant, N. Tuberculosis of the uro-genital system - urinary tract tuberculosis: renal tuberculosis. II. Indian J. Radiol. Imaging $\mathbf{2 3}$ 64-77 (2013).

109. Wei, H. L. et al. Renal tuberculosis and iliopsoas abscess: two case reports. Exp. Ther. Med. 7 1718-1720 (2014).

110. Puigvert, A. The ureter in renal tuberculosis. Br. J. Urol. 27, 258-262 (1955)

111. Friedenberg, R. M., Ney, C. \& Stachenfeld, R. A Roentgenographic manifestations of tuberculosis of ureter. J. Urol. 99, 25-29 (1968).

112. Gibson, M. S., Puckett, M. L. \& Shelly, M. E. Renal tuberculosis. Radiographics 24, 1 (2004).

113. Roylance, J., Penry, B., Rhys Davies, E. \& Roberts, M. Radiology in the management of urinary tract tuberculosis. Br. J. Urol. 42, 679-687 (1970).

114. Johnstone, A. S. Tuberculous cystitis; notes on three cases. Br. J. Radiol. 20, 61-62 (1947).

115. Kulchavenya, E. \& Cherednichenko, A. Urogenital tuberculosis, the cause of ineffective antibacterial therapy for urinary tract infections. Ther. Adv. Urol. 10, 95-101 (2017)

116. Shah, H. U., Sannananja, B., Baheti, A. D. Udare, A. S. \& Badhe, P. V. Hysterosalpingography and ultrasonography findings of female genital tuberculosis. Diagn. Interv. Radiol. 21, 10-15 (2015).

117. Singal, A., Pandhi, D., Kataria, V. \& Arora, V. K Tuberculosis of the glans penis: an important differential diagnosis of genital ulcer disease Int. J. STD AIDS 28, 1453-1455 (2017).

118. Kulchavenya, E., Brizhatyuk, E. \& Khomyakov, V. Diagnosis and therapy for prostate tuberculosis. Ther. Adv. Urol. 6, 129-134 (2014).

119. Miller, E. \& Lustok, M. Genital tuberculosis. JAMA 113, 1388-1394 (1939)

120. López Barōn, E., Gômez-Arbeláez, D. \& Díaz-Pérez, J. Primary prostatic tuberculosis. Case report and bibliographic review. Arch. Esp. Urol. 62, 309-313 (2009).

121. Sporer, A. $\&$ Oppenheimer, G. Tuberculosis of the prostate and seminal vesicles. J. Urol. 78, 278-286 (1957).
122. Stillwell, T., Engen, D. \& Farrow, G. The clinical spectrum of granulomatous prostatitis: a report of 200 cases. J. Urol. 138, 320-323 (1987)

123. Gupta, N., Mandal, A. K. \& Singh, S. K. Tuberculosis of the prostate and urethra: a review. Indian J. Urol. 24, 388-391 (2008)

124. Bouchikhi, A. A., Khallouk, A., El Fassi, M. J. \& Farih, M. H. Atypical isolated urethral tuberculosis associated with inflammatory stenosis and fistulas. Urol. Ann. 6, 270-271 (2014).

125. Hosamirudsari, H. \& Mohammadizia, F. Unilateral tuberculous epididymo-orchitis with scrotal fistula: a case report. Iran. J. Pathol. 10, 165-168 (2015).

126. Joneja, U., Short, W. R. \& Roberts, A. L. Disseminated tuberculosis with prostatic abscesses in an immunocompromised patient - a case report and review of literature. IDCases 5, 15-20 (2016).

127. Kostakopoulos, A. et al. Tuberculosis of the prostate Int. Urol. Nephrol. 30, 153-157 (1998)

128. Bour, L. et al. Multiparametric MRI features of granulomatous prostatitis and tubercular prostate abscess. Diagn. Interv. Imaging 94, 84-90 (2013).

129. Lee, I. K., Yang, W. C. \& Liu, J. W. Scrotal tuberculosis in adult patients: a 10-year clinical experience. $\mathrm{Am}$. J. Trop. Med. Hyg. 77, 714-718 (2007).

130. Borthwick, W. The pathogenesis of tuberculous epididymitis. Edin. Med. J. 53, 55-70 (1946).

131. Riehle, R. A. \& Jayraman, K. Tuberculosis of testis. Urology 1, 43-46 (1982)

132. Ross, J. C., Gow, J. G. \& St. Hill, C. A. Tuberculous epididymitis. A review of 170 patients. Br. J. Surg. 48 663-666 (1961)

133. Das, A., Batabyal, S., Bhattacharjee, S. \& Sengupta, A. A rare case of isolated testicular tuberculosis and review of literature. J. Family Med. Prim. Care. $\mathbf{5}$ 468-470 (2016)

134. Jacob, J. T., Nguyen, T. M. \& Ray, S. M. Male genital tuberculosis. Lancet Infect. Dis. 8, 335-342 (2008).

135. Madeb, R., Marshal, J., Nativ, O. \& Erturk, E. Epididymal tuberculosis: case report and review of the literature. Urology 65, 798 (2005)

136. Viswaroop, B. S., Kekre, N. \& Gopalakrishnan, G Isolated tuberculous epididymitis: a review of forty cases. J. Postgrad. Med. 51, 109-111 (2005).

137. Hadadi, A., Pourmand, G. \& Mehdipour-Aghabagher, B. Unilateral testicular tuberculosis: case report. Andrology 44, 70-72 (2012).

138. Kretschmer, H. Tuberculosis of the epididymis: a review of 170 patients. Surg. Gynecol. Obstet. 47, 652-659 (1928).

139. Heaton, N. D., Hogan, B., Michell, M., Thompson, P. $\&$ Yates-Bell, A. J. Tuberculous epididymo-orchitis: clinical and ultrasound observations. Br. J. Urol. 64 305-309 (1989).

140. Benjelloun, A., Elktaibi, A., Elharrech, Y., Touiti, D. $\&$ Ghoundale, O. Tuberculosis of the spermatic cord: case report. Urol. Case Rep. 2, 176-177 (2014).

141. Premkumar, A. \& Newhouse, J. Seminal vesicle tuberculosis: CT appearance. J. Comput. Assist. Tomogr 12, 676-677 (1988).

142. Kar, J. \& Kar, M. Primary tuberculosis of the glans penis. J. Assoc. Physicians India 60, 52-53 (2012).

143. Khan, D., Choudhary, A., Dutta, A. \& Khan, I. Tuberculosis of the glans penis mimicking as carcinoma. Int. J. Mycobacteriol. 5, 341-342 (2016).

144. Symes, J. M. \& Blandy, J. P. Tuberculosis of the male urethra. Br. J. Urol. 45, 432-436 (1973).

145. Indudhara, R., Vaidyanathan, S. \& Radotra, B. D. Urethral tuberculosis. Urol. Int. 48, 436-438 (1992).

146. Paul, J., Krishnamoorthy, S., Teresa, M. \& Kumar, S. Isolated tuberculous orchitis: a mimicker of testicular malignancy. Indian J. Urol. 26, 284-286 (2010).

147. Gangalakshmi, C. \& Sankaramahalingam, A Tuberculosis of the glans penis. J. Clin. Diagn. Res. 12 PD05-PD06 (2016).

148. Chowdhury, A. \& Dey, R. Penile tuberculosis following intravesical Bacille Calmette-Cuérin immunotherapy. Indian J. Urol. 29, 64-66 (2013).

149. Mayilvaganan, K. R., Naren Satya Srinivas, M., Reddy, V. N. \& Singh, R. K. Tuberculosis penis with 'Watering can penis' appearance: report of a rare case with retrograde urethrography and voiding cystourethrography findings. Pol. J. Radiol. 81, 454-457 (2016)

150. Buppasiri, P., Temtanakitpaisan, T. \& Somboonporn, W. Tuberculosis at vulva and vagina. J. Med. Assoc. Thai. 93, 613-615 (2010)

151. Nemati, E., Taheri, S., Nourbala, M. H. \& Einollahi, B. Vaginal tuberculosis in an elderly kidney transplant recipient. Saudi J. Kidney Dis. Transpl. 20, 465-467 (2009). 
152. Sharma, C., Shekhar, S., Sharma, V., Sharma, M. \& Aggarwal, T. Paucibacillary tubercular vulval ulcer in a sexually inactive pubertal girl: role of therapeutic trial. J. Pediatr. Adolesc. Gynecol. 25, e 123-e124 (2012).

153. Tiwari, P., Pal, D. K., Moulik, D. \& Choudhury, M. K Hypertrophic tuberculosis of vulva - a rare presentation of tuberculosis. Indian J. Tuberc $\mathbf{5 7}$, 95-97 (2010).

154. Mondal, S. K. Histopathologic analysis of female genital tuberculosis: a fifteen-year retrospective study of 110 cases in eastern India. Turk Patoloji Derg. 29 41-45 (2013)

155. Kulchavenya, E. \& Dubrovina, S. Typical and unusual cases of female genital tuberculosis. IDCases 1 , 92-94 (2014).

156. Mahajan, N., Naidu, P. \& Deep Kaur, S. Insight into the diagnosis and management of subclinical genital tuberculosis in women with infertility. J. Hum. Reprod. Sci. 9, 135-144 (2016)

157. Das, P., Ahuja, A. \& Gupta, S. D. Incidence, etiopathogenesis and pathological aspects of genitourinary tuberculosis in India: a journey revisited. Indian J. Urol. 24, 356-361 (2008)

158. Türkmen, I. C. et al. Female genital system tuberculosis: a retrospective clinicopathological study of 1,548 cases in Turkish women. Arch. Gynecol. Obstet. 286, 379-384 (2012).

159. Sharma, J. B. Current diagnosis and management of female genital tuberculosis. J. Obstet. Gynaecol. India 65, 362-371 (2015)

160. Akbulut, S., Arikanoglu, Z \& Basbug, M. Tubercular tubo-ovarian cystic mass mimicking acute appendicitis: a case report. J. Med. Case Rep. 5, 363 (2011).

161. Adsuar, N., Blanchette, H. \& Kilchevsky, E. Tuberculosis peritonitis mimicking ovarian cancer in a 20-year-old woman. A case report. J. Reprod. Med. 49, 52-54 (2004).

162. Neonakis, I. et al. Genital tuberculosis in a tamoxifen-treated postmenopausal woman with breast cancer and bloody vaginal discharge. Ann. Clin Microbiol. Antimicrob. 5, 20 (2006).

163. Shirazi, M. et al. Tuberculosis endometritis presenting as a leiomyoma. Int. J. Fertil. Steril. 8, 481-484 (2015).

164. Samal, S., Gupta, U. \& Agarwal, P. Menstrual disorders in genital tuberculosis. J. Indian Med. Assoc. 98, 126-127,129 (2000)

165. Perdhana, R. et al. Patients with secondary amenorrhea due to tuberculosis endometritis towards the induced anti-tuberculosis drug category 1. Pan Afr. Med. J. 24, 121 (2016)

166. Nezar, M. et al. Genital tract tuberculosis among infertile women: an old problem revisited. Arch. Gynecol. Obstet. 280, 787-791 (2009).

167. Singh, N., Sumana, G. \& Mittal, S. Genital tuberculosis: a leading cause for infertility in women seeking assisted conception in North India. Arch. Gynecol. Obstet. 278, 325-327 (2008).

168. Sharma, J. B. et al. Laparoscopic findings in female genital tuberculosis. Arch. Gynecol. Obstet. 278 359-364 (2008).

169. Sharma, J. B. et al. High prevalence of Fitz-HughCurtis Syndrome in genital tuberculosis. Int. J. Gynaecol. Obstet. 99, 62-63 (2007).

170. Coremans, L. \& de Clerck, F. Fitz-Hugh-Curtis syndrome associated with tuberculous salpingitis and peritonitis: a case presentation and review of literature. BMC Gastroenterol. 18, 42 (2018).

171. Singh, S. et al. Tuberculosis of uterine cervix: a report of two cases with variable clinical presentation. Trop. Doct. 40, 125-126 (2010).

172. Mukerji, S. et al. Difficulties in diagnosing tuberculosis of the cervix in a post menopausal woman: case repor and literature review. Australas. Med. J. 6, 367-370 (2013).

173. Sharma, N., Singh, A. S., Khonglah, Y. \& Mishra, J. Primary tuberculosis of cervix: a coincidental finding. J. Reprod. Infertil. 17, 247-249 (2016).

174. Rodpenpear, N. Tuberculosis of cervix resembling cervical. Cancer J. Med. Assoc. Thai. 99, S249-S252 (2016).

175. Jaiprakash, P., Pai, K. \& Rao, L. Diagnosis of tuberculous cervicitis by Papanicolaou-stained smear. Ann. Saudi Med. 33, 76-78 (2013).

176. Sabita, S., Sharmila, V., Arun Babu, T., Sinhasan, S. \& Darendra, S. A rare case of cervical tuberculosis which simulated carcinoma of the cervix. J. Clin. Diagn. Res. 7. 1189-1190 (2013)

177. Nanjappa, V. et al. Vulval tuberculosis - an unusual presentation of disseminated tuberculosis. J. Assoc. Physicians India 60, 49-52 (2012).
178. Mallya, V., Yadav, Y. K. \& Gupta, K. Vulval tuberculosis masquerading as vulval carcinoma. J. Postgrad. Med. 58, 307-308 (2012)

179. Wang, J., Fan, S., Xiao, J. \& Liang, C.-Z. Renal tuberculosis tends to be low symptoms: how to improve the diagnosis and treatment of renal tuberculosis. Asian J. Androl 18, 145-146 (2016).

180. Wise, G. J. \& Marella, V. K. Uro-genital manifestations of tuberculosis. Urol. Clin. N. Am. 30, 111-121 (2003).

181. Kapoor, R., Ansari, M. S., Mandhani, A. \& Gulia, A. Clinical presentation and diagnostic approach in cases of uro-genital tuberculosis. Indian J. Urol. 24 401-405 (2008)

182. Zarrabi, A. D. \& Heyns, C. F. Clinical features of confirmed versus suspected urogenital tuberculosis in region with extremely high prevalence of pulmonary tuberculosis. Urology 74, 41-45 (2009).

183. Kulchavenya, E. \& Kholtobin, D. Diseases masking and delaying the diagnosis of urogenital tuberculosis. Ther. Adv. Urol. 7, 331-338 (2015).

184. Bacci, M. R., Namura, J. J. \& Lera, A. T. Complicated urinary infection and extrapulmonary tuberculosis. BMJ Case Rep. 2012, bcr2012007553 (2012).

185. Kulchavenya E., Kholtobin D., Shevchenko S. Challenges in urogenital tuberculosis. World J. Urol. https://doi.org/10.1007/s00345-019-02767-x (2019).

186. Waikhom, R., Sarkar, D., Bennikal, M. \& Pandey, R. Rapidly progressive glomerulonephritis in tuberculosis. Saudi J. Kidney Dis. Transpl. 25, 872-875 (2014)

187. Kanodia, K. V. Vanikar, A. V., Patel, R. D., Suthar, K. S. \& Trivedi, H. L. Crescentic glomerulonephritis associated with pulmonary tuberculosis. J. Clin Diagn. Res. 10, ED01-ED02 (2016).

188. Shang, M. H. et al. Membranous nephropathy associated with tuberculosis. Chin. Med. J. (Engl). 129, 622-623 (2016).

189. Çakar, B. $\&$ Çiledağ, A. Evaluation of coexistence of cancer and active tuberculosis; 16 case series. Respir. Med. Case Rep. 23, 33-37 (2017).

190. Malik, R., Pathak, N. \& Sharma, S. Secondary renal amyloidosis in pulmonary tuberculosis - a classic revisited. J. Case Rep. Stud. 5, 404 (2017).

191. Bouziane, Z. et al. Tuberculosis of the renal artery: a rare cause of renovascular arterial hypertension Ann. Vasc. Surg. 23, 786.e7-786.e9 (2009)

192. Kulchavenya, E. Best practice in the diagnosis and management of urogenital tuberculosis. Ther. Adv. Urol. 5, 143-151 (2013).

193. Trauzzi, S J. et al. Management of prostatic abscess in patients with human immunodeficiency syndrome Urology 43, 629-633 (1994).

194. Aziz, E. M., Abdelhak, K. \& Hassan, F. M. Tuberculous prostatitis: mimicking a cancer. Pan Afr. Med. J. 25 130 (2016).

195. Suankwan, U. et al. A clinicopathologic study of tuberculous epididymo-orchitis in Thailand. Southeast Asian J. Trop. Med. Publ. Health 43, 951-958 (2012).

196. Kulchavenya, E., Kim, C. S., Bulanova, O. \& Zhukova, I. Male genital tuberculosis: epidemiology and diagnostic. World J. Urol. 30, 15-21 (2012)

197. Yang, D. M. et al. Comparison of tuberculous and pyogenic epididymal abscesses: clinical, gray-scale sonographic, and color doppler sonographic features. AJR Am. J. Roentgenol. 177, 1131-1135 (2001).

198. Pryor, J. P. \& Hendry, W. F. Ejaculatory duct obstruction in subfertile males: analysis of 87 patients. Fertil. Steril. 65, 725-730 (1991).

199. Agarwal, A., Kumar, N. \& Kishore, K. Evaluation of manual Mycobacterium growth indicator tube for isolation and susceptibility testing of Mycobacterium tuberculosis for implementation in low and medium volume laboratories. Med. J. Armed Forces India 74 , 220-226 (2018).

200. Prakash G et al. Primary tuberculosis of urethra presenting as stricture urethra and watering can perineum: a rarity. Urol. Ann. 8, 493-495 (2016)

201. Ilmer, M., Bergauer, F., Friese, K. \& Mylonas, I. Genital tuberculosis as the cause of tuboovarian abscess in an immunosuppressed patient. Infect. Dis. Obstet. Gynecol. 2009, 745060 (2009).

202. Romaniuk, A. et al. A rare case of tuberculous salpingitis. Interv. Med. Appl. Sci. 8, 131-134 (2016).

203. Nkhili, H., Abdelhak, M. \& Benhmamouch, M. N Pelvic tuberculosis simulating ovarian cancer in children about a case. Pediatrie Pratique http://www pediatrie-pratiquecom/journal/article/chez-lenfantpropos-dun (2012)
204. Lantheaume, S. et al. Peritoneal tuberculosis simulating advanced ovarian carcinoma: a case report. Gynecol. Obstet. Fertil. 31, 624-626 (2003).

205. Djuwantono, T. et al. Female genital tuberculosis and infertility: serial cases report in Bandung, Indonesia and literature review. BMC Res. Notes 10, 683 (2017).

206. Sharma, J. B. et al. Genital tuberculosis: an important cause of Asherman's syndrome in India. Arch. Gynecol. Obstet. 277, 37-41 (2008)

207. Gupta, B., Shree, S., Rajaram, S. \& Goel, N Genital tuberculosis: unusual presentations. Int. J. Mycobacteriol. 3, 357-359 (2016).

208. Koller, A. B. Granulomatous lesions of the cervix uteri in Black patients. S. Afr. Med. J. 49, 1228-1232 (1975).

209. Gupta, R., Dey, P., Jain, V. \& Gupta, N. Cervical tuberculosis detection in Papanicolaou-stained smear: case report with review of literature. Diagn. Cytopathol. 37, 592-595 (2009).

210. Paudel, V., Chudal, D. \& Pokhrel, D. B. Tuberculosis and HIV co-infection; the deadly duos in vulva. Indian J. Tuberc. 65, 277-279 (2018).

211. Gilpin, C., Korobitsyn, A., Migliori, G. B., Raviglione, M. C. $\&$ Weyer, K. The World Health Organization standards for tuberculosis care and management. Eur. Respir. J. 51, 1800098 (2018)

212. Lawn, S. D. et al. Advances in tuberculosis diagnostics the Xpert MTB/RIF assay and future prospects for a point-of-care test. Lancet Infect. Dis. 13, 349-361 (2013).

213. Walzl, G. et al. Tuberculosis: advances and challenges in development of new diagnostics and biomarkers. Lancet Infect. Dis. 18, e199-e210 (2018).

214. Hongler, J. et al. Comparison of Löwenstein-Jensen and BACTEC MGIT 960 culture for Mycobacterium tuberculosis in people living with HIV. HIV Med. 19 654-661 (2018)

215. Davis, J. L., Cattamanchi, A., Cuevas, L. E., Hopewell, P. C. \& Steingart, K. R. Diagnostic accuracy of same-day microscopy versus standard microscopy for pulmonary tuberculosis: a systematic review and meta-analysis. Lancet Infect. Dis. 13, 147-154 (2013).

216. Bhalla, M. et al. Performance of light-emitting diode fluorescence microscope for diagnosis of tuberculosis. Int. J. Mycobacteriol. 2, 174-178 (2013).

217. Colabawalla, B. N. Reflections on urogential tuberculosis. Indian J. Urol. 6, 51-59 (1990).

218. Berta, M. et al. Bacteriological diagnosis of renal tuberculosis: an experience at the regional tuberculosis laboratory in Córdoba Province. Argentina. Rev. Argentina Microbiología 43, 191-194 (2011).

219. Hemal, A. K. et al. Polymerase chain reaction in clinically suspected genitourinary tuberculosis: comparison with intravenous urography, bladder biopsy, and urine acid fast bacilli culture. Urology 56 570-574 (2000)

220. McNerney, R. \& Zumla, A. Impact of the Xpert MTB RIF diagnostic test for tuberculosis in countries with a high burden of disease. Curr. Opin. Pulm. Med. 21 , 304-308 (2015)

221. Tortoli, E., Benedetti, M., Fontanelli, A. \& Simonetti, M. T. Evaluation of automated BACTEC MGIT 960 system for testing susceptibility of Mycobacterium tuberculosis to four major antituberculous drugs: comparison with the radiometric BACTEC 460TB method and the agar plate method of proportion. J. Clin. Microbiol. 40, 607-610 (2002).

222. World Health Organization. Policy statement automated real-time nucleic acid amplification technology for rapid and simultaneous detection of tuberculosis and rifampicin resistance: Xpert MTB/RIF system (WHO, Geneva, 2011).

223. Bates, M. \& Zumla, A. The development. Evaluation and performance of molecular diagnostics for detection of Mycobacterium tuberculosis. Expert Rev. Mol. Diagn. 16, 307-322 (2016).

224. Green, C. et al. Rapid diagnosis of tuberculosis through the detection of mycobacterial DNA in urine by nucleic acid amplification methods. Lancet Infect. Dis. 9, 505-511 (2009).

225. Atherton, R. R. et al. Detection of Mycobacterium tuberculosis in urine by Xpert MTB/RIF Ultra: a useful adjunctive diagnostic tool in HIV-associated tuberculosis. Int. J. Infect. Dis. 75, 92-94 (2018).

226. Babafemi, E. O. et al. Complicated urinary infection and extrapulmonary tuberculosis. BMJ Case Rep. 2012, bcr2012007553 (2012).

227. Pang, Y. et al. GeneXpert MTB/RIF assay in the diagnosis of urinary tuberculosis from urine specimens. Sci. Rep. 7, 6181 (2017). 
228. Gupta-Wright, A. et al. Rapid urine-based screening for tuberculosis in HIV-positive patients admitted to hospital in Africa (STAMP): a pragmatic, multicentre parallel-group, double-blind, randomised controlled trial. Lancet 392, 292-301 (2018).

229. WHO. The use of next-generation sequencing technologies for the detection of mutations associated with drug resistance in Mycobacterium tuberculosis complex: technical guide. WHO https://apps. who.int iris/bitstream/handle/10665/274443/WHO-CDS-TB2018.19-eng.pdf (2018)

230. Lawn, S. D. \& Gupta-Wright, A. Detection of lipoarabinomannan (LAM) in urine is indicative of disseminated TB with renal involvement in patients living with HIV and advanced immunodeficiency: evidence and implications. Trans. R. Soc. Trop. Med. Hyg. 110, 180-185 (2016).

231. Colijn, C. \& Cohen, T. Whole-genome sequencing of Mycobacterium tuberculosis for rapid diagnostics and beyond. Lancet Respir. Med. 4, 6-8 (2016).

232. Doyle, R. M. et al. Direct whole-genome sequencing of sputum accurately identifies drug-resistant Mycobacterium tuberculosis faster than MGIT culture sequencing. J. Clin. Microbiol. 56, e00666-18 (2018).

233. Trașcă, E., Trașcă, E. T., Buzulică, R., Drăgoi, G. \& Nicolescu, I. The place and the role of histological examination in diagnostic algorithm of urinary system tuberculosis. Rom. J. Morphol. Embryol. 46, 105-108 (2005)

234. Kulchavenya, E. \& Khomyakov, V. Male genital tuberculosis in Siberians. World J. Urol. 24, 74-78 (2006).

235. Viswaroop, B. et al. Fine-needle aspiration cytology versus open biopsy for evaluation of chronic epididymal lesions: a prospective study. Scand. J. Urol. Nephrol. 39, 219-221 (2005).

236. Sah, S. P., Bhadani, P. P., Regmi, R., Tewari, A. \& Raj, G. A. Fine needle aspiration cytology of tubercula epididymits and epididymo-orchitis. Acta Cytol. 50, 243-249 (2006)

237. Kumar, P., Owji, S. \& Kherzi, A. Tuberculous orchitis diagnosed by fine needle aspiration cytology. Acta Cytol. 40, 1253-1256 (1996).

238. Comiter, C. V. et al. Nonpalpable intratesticula masses detected sonographically. J. Urol. 154, 1367-1369 (1995).

239. Cek, M. et al. EAU guidelines for the management of genitourinary tuberculosis. Eur. Urol. 48, 353-362 (2005).

240. Valentini, A. L., Summaria, V. \& Marano, P. Diagnostic imaging of uro-genital tuberculosis. Rays $\mathbf{2 3}$, 126-143 (1998)

241. Muttarak, M., ChiangMai, W. N. \& Lojanapiwat, B. Tuberculosis of the uro-genital tract: imaging features with pathological correlation. Singapore Med. J. 46 568-574 (2005)

242. Jung, Y. Y., Kim, J. K. \& Cho, K. S. Uro-genital tuberculosis: comprehensive cross-sectional imaging AJR Am. J. Roentgenol. 184, 143-150 (2005).

243. Tirumani, S. H. et al. Imaging of tuberculosis of the abdominal viscera: beyond the intestines. J. Clin. Imag. Sci. 3, 17 (2013).

244. Kollins, S. A., Hartman, G. W., Carr, D. T., Segura, J. W. $\S$ Hattery, R. R. Roentgenographic findings in urinary tract tuberculosis. A 10-year review. Am. J. Roentgenol. Radium Ther. Nucl. Med. 121, 487-499 (1974).

245. Dyer, R. B., Chen, M. Y. \& Zagoria, R. J. Abnormal calcifications in the urinary tract. Radiographics 18 1405-1424 (1998)

246. Vijayaraghavan, S. B. et al. Spectrum of highresolution sonographic features of urinary tuberculosis. J. Ultrasound Med. 23, 585-594 (2004).

247. Rui, X., Li, X. D., Cai, S., Chen, G. \& Cai, B. Ultrasonographic diagnosis and typing of renal tuberculosis. Int J. Urol 15, 135-139 (2008).

248. Hamrick-Turner, J., Abbitt, P. L. \& Ros, P. R. Tuberculosis of the lower uro-genital tract: findings on sonography and MR. AJR Am. J. Roentgenol. 158 919 (1992).

249. Engin, G., Acunas, B., Acunas, G. \& Tunaci, M. Imaging of extrapulmonary tuberculosis. Radiographics 20, 471-488 (2000)

250. Chung, J. J., Kim, M.-J., Lee, T., Yoo, H. S. \& Lee, J. T. Sonographic findings in tuberculous epididymitis and epididymo-orchitis. J. Clin. Ultrasound 25, 390-394 (1997).

251. Awasthi, S., Saxena, M., Ahmad, F., Kumar, A $\&$ Dutta, S. Abdominal tuberculosis: a diagnostic dilemma. J. Clin. Diagn. Res. 9, EC01-EC03 (2015).
252. Navarro-Vilasaró, M. et al. Genitourinary mycobacteriosis: retrospective study of 45 cases in a general hospital. Enferm. Infecc. Microbiol. Clin. 9 540-545 (2008)

253. Sallami, S. et al. Imaging findings of urinary tuberculosis on computerized tomography versus excretory urography: through 46 confirmed cases. Tunis. Med. 12, 743-747 (2014).

254. Kenney, P. J. Imaging of chronic renal infections. Am. J. Roentgenol. 3, 485-494 (1990)

255. Gaudiano, C. et al. Multidetector CT urography in urogenital tuberculosis: use of reformatted images for the assessment of the radiological findings. A pictorial essay. Abdom. Radiol. 9, 2314-2324 (2017).

256. Goel, A. \& Dalela, D. Options in the management of tuberculous ureteric stricture. Indian J. Urol. 24 376-381 (2008)

257. Aswathaman, K. \& Devasia, A. Thimble bladder. ANZ J. Surg. 78, 1049 (2008).

258. Wang, L.-J., Wong, Y.-C., Chen, C.-J. \& Lim, K.-E. CT features of genitourinary tuberculosis. J. Comput. Assist. Tomogr. 21, 254-258 (1997).

259. Lu, P., Li, C. \& Zhou, X. Significance of the CT scan in renal tuberculosis. Zhonghua Jie He He Hu Xi Za Zhi 24, 407-409 (2001).

260. Wang, L. J. et al. Imaging findings of urinary tuberculosis on excretory urography and computerized tomography. J. Urol. 169, 524-528 (2003).

261. Sinan, T., Sheikh, M., Ramadan, S., Sahwney, S. $\delta$ Behbehani, A. CT features in abdominal tuberculosis: 20 years' experience. BMC Med. Imaging 2, 3 (2002)

262. Kukrej, N., Cook, G. J. \& Pattison, J. M. Positron-emission tomography used to diagnose tuberculosis in a renal transplant patient. Am. J. Transplant. 2, 105-107 (2002).

263. Mahajan, M. S., Bedmutha, A. \& Singh, N. 18F-fludeoxyglucose positron emission tomography computed tomography-guided diagnosis of prostati and leptomeningeal tuberculosis. Indian J. Urol. 33 325-327 (2017)

264. Coleman, M. T. et al. Early changes by (18) fluorodeoxyglucose positron emission tomography co-registered with computed tomography predict outcome after Mycobacterium tuberculosis infection in cynomolgus macaques. Infect. Immun. 82 2400-2404 (2014).

265. Martin, C. et al. Prospective serial FDG PET/CT during treatment of extrapulmonary tuberculosis in HIV-infected patients: an exploratory study. Clin. NuCl. Med. 43, 635-640 (2018).

266. Gambhir, S. et al. Imaging in extrapulmonary tuberculosis. Int. J. Infect. Dis. 56, 237-247 (2017).

267. Subramanyam, P. \& Palaniswamy, S. S. Dual time point (18)F-FDG PET/CT imaging identifies bilateral renal tuberculosis in an immunocompromised patient with an unknown primary malignancy. Infect. Chemother. 47, 117-119 (2015).

268. da Rocha, E. L. et al. Abdominal tuberculosis: a radiological review with emphasis on computed tomography and magnetic resonance imaging findings. Radiol. Bras. 48, 181-191 (2015).

269. Rais-Bahrami, S et al Clinical and multiparametric MRI signatures of granulomatous prostatitis. Abdom Radiol. 42, 1956-1962 (2017).

270. Cheng, Y., Huang, L., Zhang, X., Ji, Q. \& Shen, W. Multiparametric magnetic resonance imaging characteristics of prostate tuberculosis. Korean $\mathrm{J}$. Radiol. 16, 846-852 (2015).

271. Liao, L. G. et al. Etiology of 305 cases of refractory hematospermia and therapeutic options by emerging endoscopic technology. Sci. Rep. 9, 5018 (2019).

272. Ahmadi, F., Zafarani, F. \& Shahrzad, G Hysterosalpingographic appearances of female genita tract tuberculosis. I. Fallopian tube. Int. J. Fertil. Steril. 7, 245-252 (2014)

273. Ahmadi, F., Zafarani, F. \& Shahrzad, G. S Hysterosalpingographic appearances of female genital tract tuberculosis. II. Uterus. Int. J. Fertil. Steril. 8 13-20 (2014).

274. Farrokh, D., Layegh, P., Afzalaghaee, M., Mohammadi, M. \& Fallah Rastegar, Y. Hysterosalpingographic finding in women with genital tuberculosis. Iran. J. Reprod. Med. 13, 297-304 (2015).

275. Maynard-Smith, L., Fernando, B., Hopkins, S., Harber, M $\&$ Lipman, M. Managing latent tuberculosis in UK renal transplant units: how does practice compare with published guidance? Clin. Med. 14, 26-29 (2014).

276. Shu, C. C. et al. Predictors and prevalence of latent tuberculosis infection in patients receiving long-term hemodialysis and peritoneal dialysis. PLOS ONE 7, e42592 (2012).
277. Hadaya, K. et al. Contribution of interferon- $\gamma$ release assays (IGRAs) to the diagnosis of latent tuberculosis infection after renal transplantation. Transplantation 95, 1485-1490 (2013).

278. WHO. Latent TB infection: updated and consolidated guidelines for programmatic management. $W H O$ https://www who int/tb/publications/2018/ latent-tuberculosis-infection/en/ (2018).

279. British Thoracic Society. Guidelines for the prevention and management of Mycobacterium tuberculosis infection and disease in adult patients with chronic kidney disease BTS Guideline Group on behalf of The British Thoracic Society Standards of Care Committee and Joint Tuberculosis Committee. Thorax 65, 559-570 (2010)

280. WHO. WHO consolidated guidelines on drug-resistant tuberculosis treatment. WHO https://www.who.int/ tb/publications/2019/consolidated-guidelines-drugresistant-TB-treatment/en/ (2019).

281. Tiberi, S. et al. Tuberculosis: progress and advances in development of new drugs, treatment regimens, and host-directed therapies. Lancet Infect. Dis. 18 e183-e198 (2018)

282. World Health Organization. Improving the diagnosis and treatment of smear-negative pulmonary and extrapulmonary tuberculosis among adults and adolescents. WHO https://www.who.int/tb/ publications/2006/tbhiv_recommendations.pdf (2006).

283. Sharma, J. B. et al. Six months versus nine months anti-tuberculous therapy for female genital tuberculosis: a randomized controlled trial. Eur. $J$. Obstet. Gynecol. Reprod. Biol. 203, 264-273 (2016).

284. Wagaskar, V. G. et al. Urinary tuberculosis with renal failure: challenges in management. J. Clin. Diagn. Res. 10, PC01-PC03 (2016).

285. Chang, C. H. et al. Acute kidney injury due to anti-tuberculosis drugs: a five-year experience in an aging population. BMC Infect. Dis. 14, 23 (2014).

286. Fischer, M. \& Flamm, J. The value of surgical therapy in the treatment of urogenital tuberculosis. Urologe $A$ 29, 261-264 (1990)

287. Carl, P. \& Stark, L. Indications for surgical management of genitourinary tuberculosis. World $\mathrm{J}$. Surg. 21, 505-510 (1997).

288. Bansal, P. \& Bansal, N. The surgical management of urogenital tuberculosis our experience and long-term follow-up. Urol. Ann. 7, 49-52 (2015).

289. Li, X. et al. A clinical comparative analysis of retroperitoneal laparoscopic tuberculous nephrectomy and open tuberculous nephrectomy. J. Laparoendosc. Adv. Surg. Tech. A 29, 909-913 (2019).

290. Gupta, R. et al. Laparoscopic ablative and reconstructive surgeries in uro-genital tuberculosis JSLS 18, e2014.00203 (2014).

291. O'Flynn, D. Surgical treatment of genito-urinary tuberculosis: a report on 762 cases. Br. J. Urol. 42 667-671 (1970)

292. Mochalova, T. P. \& Starikov, I. Y. Reconstructive surgery for treatment of urogenital tuberculosis: 30 years of observation. World J. Surg. 21, 511-515 (1997)

293. Zwergel, U., Wullich, B., Rohde, V. \& Zwergel, T Surgical management of urinary tuberculosis: a review of 341 patients. J. Urol. 161, 9 (1999).

294. Krishnamoorthy, S. \& Gopalakrishnan, G. Surgical management of renal tuberculosis. Indian J. Urol. 24 369-375 (2008).

295. Rizzo, M. et al. Twenty-years experience on genitourinary tuberculosis. Arch. Ital. Urol. Androl. 76, 83-87 (2004).

296. Mcaleer, S. J., Johnson, C. W. \& Johnson, W. D. in Campbell-Walsh Urology 9th edn (ed. Wein, A. J.) 436-470 (Saunders Elsevier, 2007).

297. Gow, J. G. Renal calcification in genitourinary tuberculosis. Br. J. Surg. 52, 283-288 (1965)

298. Kim, H. H., Lee, K. S., Park, K. \& Ahn, H. Laparoscopic nephrectomy for nonfunctioning tuberculous kidney. J. Endourol. 14, 433-437 (2000).

299. Rassweiler, J. et al. Laparoscopic nephrecthe: the experience of the laparoscopy working group of the German Urologic Association. J. Urol. 160, 18-21 (1998).

300. Gupta, S. et al. Acute renal failure in bilateral urinary tract tuberculosis. Pediatr. Surg. Int. 13, 200-201 (1998).

301. Vasdev, N., Moon, A.\& Thorpe, A. C. Metabolic complications of urinary intestinal diversion. Indian J. Urol. 29, 310-315 (2013).

302. Gupta, R., Singh, P. \& Kumar, R. Should men with 
genitourinary tuberculosis? J. Hum. Reprod. Sci. 8 , 43-47 (2015).

303. Nickel, J. C. Chronic epididymitis: a practical approach to understanding and managing a difficult urologic enigma. Rev. Urol. 5, 209-215 (2003).

304. Cho, Y. S., Joo, K. J., Kwon, C. H. \& Park, H. J. Tuberculosis of testis and prostate that mimicked testicular cancer in young male soccer player. J. Exerc. Rehabil. 9, 389-393 (2013)

305. Weiner, J. 3rd et al. Metabolite changes in blood predict the onset of tuberculosis. Nat. Commun. 9 5208 (2018).

306. Duffy, F. J. et al. Immunometabolic signatures predict risk of progression to active tuberculosis and disease outcome. Front. Immunol. 10, 52 (2019).

307. Fitzgerald, B. L. et al. Elucidation of a human urine metabolite as a seryl-leucine glycopeptide and as a biomarker of effective anti-tuberculosis therapy. ACS Infect. Dis. 5, 353-364 (2019).

308. MacLean, E. et al. A systematic review of biomarkers to detect active tuberculosis. Nat. Microbiol. 4 748-758 (2019).

309. Zumla, A. et al. Host-directed therapies for infectious diseases: current status, recent progress, and future prospects. Lancet Infect. Dis. 16, e47-e63 (2016).

310. Liu, C. et al. Quantification of circulating Mycobacterium tuberculosis antigen peptides allows rapid diagnosis of active disease and treatment monitoring. Proc. Natl. Acad. Sci. USA 114 3969-3974 (2017).

311. Rao, M. et al. Improving treatment outcomes for MDR-TB - novel host-directed therapies and personalised medicine of the future. Int. J. Infect. Dis. 80S, S62-S67 (2019).

312. Skrahin, A. et al. Autologous mesenchymal stromal cell infusion as adjunct treatment in patients with multidrug and extensively drug-resistant tuberculosis: an open-label phase 1 safety trial. Lancet Respir. Med. 2, 108-122 (2014).

313. Yudintceva, N. M. et al. Application of the allogenic mesenchymal stem cells in the therapy of the bladder tuberculosis. J. Tissue Eng. Regen. Med. 12 e1580-e1593 (2018).

314. Kadhiravan, T. \& Sharma, S. K. Medical management of genitourinary tuberculosis. Indian J. Urol. $\mathbf{2 4}$, 362-368 (2008)

315. WHO. Guidelines on the management of latent tuberculosis infection. WHO https://www.who. int/tb/publications/latent-tuberculosis-infection/en/ (2019).

316. White, R. G., Hanekom, W. A., Vekemans, J. \& Harris, R. C. The way forward for tuberculosis vaccines. Lancet Respir. Med. 7, 204-206 (2019).

317. Zumla, A. \& Petersen, E. The historic and unprecedented United Nations General Assembly High Level Meeting on Tuberculosis (UNGA-HLM-TB) - United to End TB: An Urgent Global Response to a Global Epidemic'. Int. J. Infect. Dis. 75, 118-120 (2018).

\section{Acknowledgements}

A.Z. receives a UK National Institutes of Health Research (NIHR) senior investigator award. A.Z. acknowledges support from the PANDORA-ID-NET grant from the EDCTP Reg/Grant RIA2016E-1609), CANTAM2, TESA2 and EACCR2 EDCTP Networks of Excellence grants, all funded by the European and Developing Countries Clinical Trials Partnership (EDCTP2) programme, which is supported under Horizon 2020, the European Union's Framework Programme for Research and Innovation. A.Z. and A.M. acknowledge support from the NIHR Biomedical Research Centre at UCLH. Figures 3a, 3b, 3c, 3d, 3i, 7c, 7d and 7e were kindly provided by Professo Sebastian Lucas, St Thomas's Hospital, London.

\section{Author contributions}

All authors researched data for the article, wrote the manuscript and reviewed and edited the manuscript before submission. B.M., S.K. and A.Z. made substantial contributions to discussions of content.

\section{Competing interests}

A.Z. serves on the $\mathrm{WHO}$ and other global tuberculosis (TB) expert advisory groups and committees and is editor of two textbooks on TB, which were used as references. The other authors declare no competing interests.

\section{Peer review information}

Nature Reviews Urology thanks E. Kulchavenya and the other, anonymous, reviewer(s) for their contribution to the peer review of this work.

\section{Publisher's note}

Springer Nature remains neutral with regard to jurisdictional claims in published maps and institutional affiliations.

\section{Review criteria}

We reviewed publications in English on the MEDLINE, EMBASE and GOOGLE SCHOLAR for the period up to 1 September 2018 using the search terms "tuberculosis" or "TB" in combination with the terms "urological", "genital", "uro-genital", "epididymo-orchitis", "kidney", or "renal" or "ureter" or "bladder" or "testes" or "urethra" or "penis" or "infertility"; websites of global and national public health agencies such as WHO, US-Centres for Disease Control, UK-Public Health England, European Centre for Disease Prevention and Control (ECDC); The 2018 WHO Global TB Report; specialist textbooks on tuberculosis; clinical guidelines developed by specialist societies; and relevant substantive reviews to inform readers of more references. 